\title{
Weighted Error Estimates for Transient Transport Problems Discretized Using Continuous Finite Elements with Interior Penalty Stabilization on the Gradient Jumps
}

\section{Erik Burman ${ }^{1}$ iD \\ Received: 14 April 2021 / Accepted: 8 October 2021 / Published online: 8 March 2022}

(C) The Author(s) 2022

\begin{abstract}
In this paper we consider the semi-discretization in space of a first order scalar transport equation. For the space discretization we use standard continuous finite elements with a stabilization consisting of a penalty on the jump of the gradient over element faces. We recall some global error estimates for smooth and rough solutions and then prove a new local error estimate for the transient linear transport equation. In particular we show that for the stabilized method the effect of non-smooth features in the solution decay exponentially from the space time zone where the solution is rough so that smooth features will be transported unperturbed. Locally the $L^{2}$-norm error converges with the expected order $O\left(h^{k+\frac{1}{2}}\right)$, if the exact solution is locally smooth. We then illustrate the results numerically. In particular we show the good local accuracy in the smooth zone of the stabilized method and that the standard Galerkin fails to approximate a solution that is smooth at the final time if underresolved features have been present in the solution at some time during the evolution.
\end{abstract}

Keywords Continuous Galerkin - Stability · Scalar hyperbolic transport equations · Initial-boundary value problem $\cdot$ Stabilized methods

Mathematics Subject Classification (2010) 65M12 - 65M20 · 65M15

\section{Introduction}

The discretization of transport problems has traditionally been dominated by discontinuous Galerkin methods or finite volume methods, typically of low order, since the continuous Galerkin method is known to have robustness problems for first order partial differential

Dedicated to Professor Alfio Quarteroni on his 70th birthday.

Erik Burman

e.burman@ucl.ac.uk

1 Department of Mathematics, University College London,

Gower Street, London, UK-WC1E 6BT, UK 
equations (see [23, Chapter 5]), or convection-diffusion equations in the convection dominated regime. In certain situations the use of high order continuous Galerkin methods is appealing, for instance in the case of convection-diffusion equations, in particular where the diffusion is nonlinear, or more complex situations such as large eddy simulation of turbulent flows, where the pressure-velocity coupling can be decoupled using a pressure projection method and the convective part handled explicitly. In such situations, if continuous finite element spaces are used, one must resort to a stabilized method to avoid a reduction of accuracy due to spurious oscillations. There is a very wide literature on stabilized methods and for an overview of the topic see for example [24]. In the high order case, the Spectral Vanishing Velocity method has been a popular choice [34-36], but other methods have also been designed to work for high order, see the discussion in [17]. In this work we will focus on the continuous interior penalty (CIP) stabilization, that was shown to allow for close to $h p$-optimal error estimates in the high Peclet regime in [10]. Recently [37] this method was applied to under resolved simulations of turbulent flows using high order polynomial approximation and shown to perform very well in this context. Therein an eigenanalysis was performed which showed that the CIP finite element method has similar advantageous dispersion properties as the discontinuous Galerkin method (see also the report [19]) and in the computations it was verified that its numerical dissipation was less important than that of the spectral vanishing viscosity.

Ideally stability of the finite element method should match that of the continuous problem. This is typically, by and large, true for elliptic pde, but much harder to achieve in the hyperbolic case. Indeed, this would mean satisfaction of a discrete maximum principle and stability and error estimates in $L^{1}$. Both which typically remain open questions. Herein we will only consider the stability in the $L^{2}$-norm for continuous finite element approximations and linear symmetric stabilization of gradient penalty type applied to the transient scalar, linear first order equation. The analysis will mainly focus on semi discretization in space on periodic domains, but the extension to the fully discrete case and weakly imposed boundary conditions will be sketched. The classical estimate for smooth solutions that is proven for stabilized finite element methods is on the form

$$
\left\|\left(u-u_{h}\right)(\cdot, T)\right\|_{\Omega} \leq C(u) h^{k+\frac{1}{2}},
$$

where $C(u)$ is a constant that depends on Sobolev norms of the exact solution and on equation data, $h$ is the mesh-size and $k$ the polynomial order. This estimate that is suboptimal by $h^{\frac{1}{2}}$ is known to be sharp on general meshes [38] (see also [7] for the sharpness of the estimate for the CIP method). The continuous Galerkin method without stabilization, however, only admits a bound of order $h^{k}$. The lost factor $h^{\frac{1}{2}}$ is of little consequence for smooth solutions, and high polynomial order. However for low polynomial order or rough solutions it becomes significant. In Section 4 below, we prove this type of error estimate and some variations in weak norm for rough solutions. This analysis uses ideas from [9, 12]. Some remarks on the time discretization will be added in Section 4.2. In particular we will point out the situations where the stabilization actually improves the stability of time stepping methods.

The estimate (1.1) is a weak result, but it has become a proxy for stronger estimates that give convergence also of the material derivative (see [13, 27] and Theorem 2 below) and importantly, local estimates, using weighted norms, well known in the stationary case 
$[14,28,32,33]$. In the context of time dependent problems such a weighted estimate takes the form

$$
\left\|\varpi\left(u-u_{h}\right)(\cdot, T)\right\|_{\Omega} \leq C h^{k+\frac{1}{2}}\left(\int_{0}^{T}\left\|\varpi D^{k+1} u\right\|_{\Omega}^{2} \mathrm{~d} t\right)^{\frac{1}{2}},
$$

where $D^{m}$ is a multi-index differential operator of order $m$ and the $\varpi$ is a weight function that is aligned with the characteristics and decays exponentially away from some zone of interest. This means that if $\varpi=1$ in some zone where the solution is smooth the influence of locally large derivatives and underresolution at some distance $d$ from this zone will be damped with a factor $e^{-d / \sqrt{h}}$. We prove such an estimate in Section 5 for the space semidiscretized stabilized formulation. To the best of my knowledge there are no previous such estimates for continuous finite element methods using symmetric stabilization. For earlier works on Streamline Upwind Petrov-Galerkin methods (SUPG) in this direction see [20, 44]. The approach in [44] relies strongly on the space time finite element discretisation and an additional artificial viscosity term and in [20] the authors consider the SUPG method together with a first order backward differentiation in time, on a form that can not easily be extended to higher order time-discretizations. In neither case can the arguments be applied independently of the time discretization. In this paper we apply the ideas from [14] where weighted estimates were proved for the stationary convection-diffusion equation with CIPstabilization and [16], where they were applied to an inverse boundary value problem subject to a convection-diffusion equation. The result is presented in detail for the semi-discretized case only, but the extension to standard stable time discretizations is sketched. The results can also be extended to the case of convection-diffusion equations with Neumann conditions on the outflow boundary, by straightforward addition of the diffusive terms and following the argument of [14].

In the numerical section (Section 6) we will illustrate this localization property of the error and show that it is not shared by the standard (unstabilized) Galerkin finite element method. Indeed, as we shall see, without stabilization Galerkin FEM fails to approximate even smooth solutions satisfactory in case the solution has had non-smooth features at any time during the computation. Indeed it appears that the standard Galerkin method does not propagate underresolved features of the solution with the right speed, making it impossible for the method to evacuate high frequency content from the computational domain. For the stabilized method on the other hand the weighted estimate (1.2) guarantees that smooth components of the solution are untainted by spurious high frequency content at all times, since perturbations are damped exponentially when crossing the characteristics.

\section{Model Problem and Finite Element Discretization}

We will discuss a first order hyperbolic problem in a periodic domain, $\Omega=[-L, L]^{n}$, where $n \geq 1$ is the space dimension. Let $\boldsymbol{\beta} \in C^{0}\left([0, T] ;\left[C^{m}(\bar{\Omega})\right]^{n}\right), m \geq 1$, be a periodic vector field satisfying $\nabla \cdot \boldsymbol{\beta}=0$ and consider the first order hyperbolic problem

$$
\begin{aligned}
\mathcal{L} u:=\partial_{t} u+\boldsymbol{\beta} \cdot \nabla u & =f & & \text { in }(0, T) \times \Omega, \\
u(\cdot, 0) & =u_{0} & & \text { in } \Omega .
\end{aligned}
$$

For smooth data $\boldsymbol{\beta}, u_{0}$ and $f$ there exists a unique solution by the method of characteristics, but the problem admits a unique solution also for more rough data [26]. The solution 
satisfies the following regularity estimate (a proof of this can be obtained after minor modifications of [5, Lemma 2]),

$$
\|u(t)\|_{H^{j}(\Omega)} \leq C_{\beta}\left(\|f\|_{L^{2}\left((0, T) ; H^{j}(\Omega)\right)}+\left\|u_{0}\right\|_{H^{j}(\Omega)}\right), \quad t>0, \quad j \geq 0 \text { when } m \geq j .
$$

Below we will always assume that $\boldsymbol{\beta}$ is smooth enough for (2.3) to hold. The constant $C_{\beta}$ grows exponentially in time, with coefficient dependent on the sup-norm of $\boldsymbol{\beta}$, and its derivatives of order up to $j$. Below the notation $\beta_{\infty}=\sup _{x \in \bar{\Omega}}|\boldsymbol{\beta}(x)|$ will be used. The $L^{2}$-norm over a domain $X \subset \Omega$ will be denoted by $\|\cdot\|_{X}=(\cdot, \cdot)_{X}^{\frac{1}{2}}$, where $(\cdot, \cdot)_{X}^{\frac{1}{2}}$ is the $L^{2}$-scalar product over $X$, also $\|\cdot\|_{\infty}$ will denote the norm on $C^{0}(\bar{\Omega})$.

Let $\{\mathcal{T}\}_{h}$ be a family of shape regular decompositions of $\Omega$ in simplices $S, \mathcal{T}=\{S\}$, indexed by the (uniform) mesh size $h$. Let $\mathcal{F}$ denote the set of faces of $\mathcal{T}$. $C$ will denote a generic constant that can have different value at each appearance, but is always independent of the mesh-parameter $h$. Now define the finite element space

$$
V_{h}:=\left\{v \in H_{p e r}^{1}(\Omega):\left.v\right|_{S} \in \mathbb{P}_{k}(S) \text {, for all } S \in \mathcal{T}\right\},
$$

where $\mathbb{P}_{k}(S)$ denotes the set of polynomials of degree less than or equal to $k$ on $S$ and $H_{p e r}^{1}(\Omega)$ denotes the set of periodic functions in $H^{1}$ on $\Omega$. We may then write a semidiscretization in space, for $t>0$ find $u_{h}(t) \in V_{h}$, with $u_{h}(0)=\pi_{h} u_{0}$, such that

$$
\left(\mathcal{L} u_{h}(t), v_{h}\right)_{\Omega}=F\left(v_{h}\right), \quad \forall v_{h} \in V_{h}
$$

where $F\left(v_{h}\right):=\left(f, v_{h}\right)_{\Omega}$. Above $\pi_{h}$ denotes the $L^{2}$-projection onto the finite element space $V_{h}$. For all $v \in L^{2}(\Omega), \pi_{h} v \in V_{h}$ satisfies

$$
\left(\pi_{h} v, w_{h}\right)_{\Omega}=\left(v, w_{h}\right)_{\Omega}, \quad \forall w_{h} \in V_{h} .
$$

It is well known that on locally quasi-uniform meshes the $L^{2}$-projection satisfies the approximation bound,

$$
\left\|v-\pi_{h} v\right\|_{\Omega}+h\left\|\nabla\left(v-\pi_{h} v\right)\right\|_{\Omega} \leq C h^{k+1}|v|_{H^{k+1}(\Omega)}, \quad \forall v \in H^{k+1}(\Omega) .
$$

The formulation (2.4) defines a dynamical system that admits a unique solution for $m \geq$ 0 using standard techniques. Taking $v_{h}=u_{h}$ in (2.4) and integrating in time we see that (2.4) satisfies the bound (2.3) with $j=0$

$$
\left\|u_{h}(t)\right\|_{\Omega} \leq C_{\beta}\|f\|_{L^{2}((0, T) ; \Omega)}+\left\|u_{0}\right\|_{\Omega}, \quad t>0 .
$$

Since $\nabla \cdot \boldsymbol{\beta}=0$ the bound holds with $C_{\beta}=T^{\frac{1}{2}}$. Actually a stronger results holds for the $L^{2}$-norm when the norm on $f$ is weakened. Indeed one may use that

$$
\int_{0}^{T}\left(f, u_{h}\right)_{\Omega} \mathrm{d} t+\left\|u_{0}\right\|_{\Omega}^{2} \leq \sup _{t \in(0, T)}\left\|u_{h}(t)\right\|_{\Omega}\left(\|f\|_{L^{1}\left((0, T) ; L^{2}(\Omega)\right)}+\left\|u_{0}\right\|_{\Omega}\right)
$$

to show that

$$
\sup _{t \in(0, T)}\left\|u_{h}(t)\right\|_{\Omega} \leq\|f\|_{L^{1}\left((0, T) ; L^{2}(\Omega)\right)}+\left\|u_{0}\right\|_{\Omega} .
$$

However (2.3) does not hold for $u_{h}$ for $j=1$. A natural question to ask is then if the solution to (2.4) gives any control of the derivatives. In case $f \in L^{2}((0, T) ; \Omega)$ the immediate control offered by (2.1) is $\mathcal{L} u \in L^{2}((0, T) ; \Omega)$, that is the material derivative is bounded in $L^{2}$. For (2.4) we get the corresponding bound $\pi_{h} \mathcal{L} u_{h} \in L^{2}((0, T) ; \Omega)$. Since $\mathcal{L} u_{h}$ may be discontinuous over element faces (due to the presence of derivatives in space) and $V_{h} \in C^{0}(\Omega)$, we see that $\pi_{h} \mathcal{L} u_{h} \neq \mathcal{L} u_{h}$. It follows that not even this weakest measure of derivatives of $u$ is controlled by (2.4). However since we are looking for control in a discrete 
space we can use norm equivalence on discrete spaces in the form of the inverse inequality [3, Lemma 4.5.3],

$$
\left\|\nabla u_{h}\right\|_{S} \leq C h^{-1}\left\|u_{h}\right\|_{S}, \quad \forall S \in \mathcal{T}
$$

and observing that $\partial_{t} u_{h} \in V_{h}$, we see that

$$
\left\|\mathcal{L} u_{h}\right\|_{\Omega} \leq\left\|\pi_{h} \mathcal{L} u_{h}\right\|_{\Omega}+C \beta_{\infty} h^{-1}\left\|u_{h}\right\|_{\Omega} .
$$

Combining (2.7) with the bound (2.5)

$$
\left\|\mathcal{L} u_{h}\right\|_{L^{2}((0, T) ; \Omega)} \leq\left(1+C_{\beta} h^{-1}\right)\left(\|f\|_{L^{2}((0, T) ; \Omega)}+\left\|u_{0}\right\|_{\Omega}\right) .
$$

So the constant in the control of the material derivative grows as $O\left(h^{-1}\right)$ under mesh refinement. Hence there is no improvement compared to obtaining an $H^{1}$ estimate by combining the $L^{2}$-stability of (2.5) with (2.6).

The rationale for the addition of stabilizing terms is to improve the control of derivatives of $u_{h}$. As an example of stabilization we here propose the gradient penalty term, introduced in [21] and shown to result in improved robustness and error estimates for convection dominated flows in [15],

$$
s\left(w_{h}, v_{h}\right)=\sum_{F \in \mathcal{F}}\left\langle h_{F}^{2}|\boldsymbol{\beta}| \llbracket \nabla u_{h} \rrbracket, \llbracket \nabla v_{h} \rrbracket\right\rangle_{F}
$$

where $\langle u, v\rangle_{F}=\int_{F} u v \mathrm{~d} s,\left.\llbracket \nabla v_{h} \rrbracket\right|_{F}=\left.\nabla v_{h}\right|_{F \cap \partial S_{1}} \cdot n_{1}+\left.\nabla v_{h}\right|_{F \cap \partial S_{2}} \cdot n_{2}$ for $F=\bar{S}_{1} \cap \bar{S}_{2}$ and $n_{1}$ and $n_{2}$ denote the outward pointing normals of the simplices $S_{1}$ and $S_{2}$ respectively. To reduce the amount of crosswind diffusion the $|\boldsymbol{\beta}|$ factor may be replaced by $|\boldsymbol{\beta} \cdot n|$. Define the stabilization semi norm by

$$
\left|w_{h}\right|_{s}:=s\left(w_{h}, w_{h}\right)^{\frac{1}{2}}
$$

Also recall the following inverse inequality

$$
\left|w_{h}\right|_{s} \leq C h^{-\frac{1}{2}} \beta_{\infty}^{\frac{1}{2}}\left\|w_{h}\right\|_{\Omega}, \quad \forall w_{h} \in V_{h}
$$

which is a consequence of the scaled trace inequality, [3, Theorem 1.6.6],

$$
\|v\|_{\partial S} \leq C_{S}\left(h^{-\frac{1}{2}}\|v\|_{S}+h^{\frac{1}{2}}\|\nabla v\|_{S}\right), \quad \forall v \in H^{1}(S)
$$

and (2.6).

The enhanced control of derivatives offered by this stabilization term can be expressed as

$$
\inf _{v_{h} \in V_{h}}\left\|h^{\frac{1}{2}}\left(\boldsymbol{\beta} \cdot \nabla u_{h}-v_{h}\right)\right\|_{\Omega}^{2} \leq C_{s}\left(\beta_{\infty}\left|u_{h}\right|_{s}^{2}+h\|\nabla \boldsymbol{\beta}\|_{\infty}^{2}\left\|u_{h}\right\|_{\Omega}^{2}\right) .
$$

This is an immediate consequence of the local estimate of [10, Lemma 5.3] and local approximation of $\boldsymbol{\beta}$ using lowest order Raviart-Thomas functions (for details see the discussion [13, Page 4]). In particular this implies (since $\partial_{t} u_{h} \in V_{h}$ ) that

$$
\left\|\mathcal{L} u_{h}\right\|_{\Omega} \leq C\left\|\pi_{h} \mathcal{L} u_{h}\right\|_{\Omega}+C_{s}^{\frac{1}{2}}\left(h^{-\frac{1}{2}} \beta_{\infty}^{\frac{1}{2}}\left|u_{h}\right|_{s}+\|\nabla \boldsymbol{\beta}\|_{\infty}\left\|u_{h}\right\|_{\Omega}\right) .
$$

It follows that when the finite element method has the additional stability offered by the operator $s$, the constant in the bound for $\mathcal{L} u_{h}$ will grow at the rate $O\left(h^{-\frac{1}{2}}\right)$ under mesh refinement. Therefore we propose the stabilized method, find $u_{h}(t) \in V_{h}$, with $u_{h}(0)=$ $\pi_{h} u_{0}$, such that

$$
\left(\mathcal{L} u_{h}(t), v_{h}\right)_{\Omega}+\gamma s\left(u_{h}, v_{h}\right)=F\left(v_{h}\right), \quad \forall v_{h} \in V_{h}
$$

for $\gamma>0$. Clearly for $\gamma=0$ we recover the standard Galerkin method. 
Remark 1 Although we only consider continuous FEM below all the results holds true for $\mathrm{dG}$ methods if the standard Galerkin method (without stabilization) is replaced by the standard dG method with central flux and the stabilized finite element method is replaced by the standard $\mathrm{dG}$ method with upwind flux. There is indeed a common misconception that the enhanced stability of the $\mathrm{dG}$ methods (space discretization) is due to the discontinuity of the element. The discontinuity only allows for the improved control of the material derivative if there is sufficent control on the solution jump. This can be introduced through upwind fluxes, or otherwise. Indeed it is easy to see that the upwind flux formulation is obtained from the central flux formulation by adding the following stabilization term [4]

$$
s_{u p}\left(v_{h}, w_{h}\right):=\frac{1}{2} \sum_{F \in \mathcal{F}}\left\langle\left|\boldsymbol{\beta} \cdot n_{F}\right|\left[v_{h}\right],\left[w_{h}\right]\right\rangle_{F},
$$

where $[\cdot]$ simply denotes the jump of the function over the element face $F$. In general the full jump needs to be penalized, but the minimal stabilization needed to make the $\mathrm{dG}$ method satisfy the bound (2.12) depends on the mesh geometry and the polynomial order [6, 39].

\section{Stability Estimate of the Finite Element Method}

Here we will formalize the discussion of the previous section to obtain a stability estimate that will be useful for the subsequent error analysis. First define the operator norms

$$
\|F\|_{0}:=\sup _{v_{h} \in V_{h}} \frac{\left|F\left(v_{h}\right)\right|}{\left\|v_{h}\right\|_{\Omega}} \quad \text { and } \quad\|F\|_{h}:=\sup _{v_{h} \in V_{h}} \frac{\left|F\left(v_{h}\right)\right|}{\left\|v_{h}\right\|_{\Omega}+\left|v_{h}\right|_{s}} .
$$

With these definitions the arguments discussed in the previous section may be written as follows.

Theorem 1 Let $u_{h}$ solve (2.13) with $\gamma>0$ then for all $\tau \in[0, T]$

$$
\left\|u_{h}(\tau)\right\|_{\Omega}^{2}+\gamma \int_{0}^{\tau}\left|u_{h}\right|_{s}^{2} \mathrm{~d} t \leq C_{\beta}\left(\int_{0}^{\tau}\|F\|_{h}^{2} \mathrm{~d} t+\left\|u_{h}(0)\right\|_{\Omega}^{2}\right)
$$

where $C_{\beta}=O\left(\gamma^{-1}+T\right)$.

Proof First take $v_{h}=u_{h}$ in (2.13) to obtain using the skew symmetry of the convective operator

$$
\left(\mathcal{L} u_{h}, u_{h}\right)_{\Omega}=\frac{1}{2} \frac{d}{d t}\left\|u_{h}(t)\right\|_{\Omega}^{2}
$$

and therefore after integration in time over $(0, \tau)$

$$
\begin{aligned}
\frac{1}{2}\left\|u_{h}(\tau)\right\|_{\Omega}^{2}+\gamma \int_{0}^{\tau}\left|u_{h}(t)\right|_{s}^{2} \mathrm{~d} t & \leq \frac{1}{2}\left\|u_{h}(0)\right\|_{\Omega}^{2}+\int_{0}^{\tau} F\left(u_{h}\right) \mathrm{d} t \\
& \leq \frac{1}{2}\left\|u_{h}(0)\right\|_{\Omega}^{2}+\int_{0}^{\tau}\|F\|_{h}\left(\left\|u_{h}(t)\right\|_{\Omega}+\left|u_{h}(t)\right|_{s}\right) \mathrm{d} t .
\end{aligned}
$$

Using the arithmetic-geometric inequality $a b \leq \frac{1}{2} a^{2}+\frac{1}{2} b^{2}$ it follows that $\|F\|_{h}\left(\left\|u_{h}(t)\right\|_{\Omega}+\right.$ $\left.\left|u_{h}(t)\right|_{s}\right) \leq\left(\gamma^{-1}+T\right)\|F\|_{h}^{2}+\frac{1}{2} T^{-1}\left\|u_{h}(t)\right\|_{\Omega}^{2}+\gamma \frac{1}{2}\left|u_{h}(t)\right|_{s}^{2}$ leading to

$\left\|u_{h}(\tau)\right\|_{\Omega}^{2}+\gamma \int_{0}^{\tau}\left|u_{h}(t)\right|_{s}^{2} \mathrm{~d} t \leq\left\|u_{h}(0)\right\|_{\Omega}^{2}+\left(\gamma^{-1}+T\right) \int_{0}^{\tau}\|F\|_{h}^{2} \mathrm{~d} t+\int_{0}^{\tau} T^{-1}\left\|u_{h}(t)\right\|_{\Omega}^{2} \mathrm{~d} t$. 
By Gronwall's inequality we have

$$
\begin{aligned}
\left\|u_{h}(\tau)\right\|_{\Omega}^{2} & \leq\left(\exp \int_{0}^{\tau} T^{-1} \mathrm{~d} t\right)\left(\left\|u_{h}(0)\right\|_{\Omega}^{2}+\left(\gamma^{-1}+T\right) \int_{0}^{\tau}\|F\|_{h}^{2} \mathrm{~d} t\right) \\
& \leq C\left(\left\|u_{h}(0)\right\|_{\Omega}^{2}+\left(\gamma^{-1}+T\right) \int_{0}^{\tau}\|F\|_{h}^{2} \mathrm{~d} t\right) .
\end{aligned}
$$

We may then bound

$$
\begin{aligned}
\gamma \int_{0}^{\tau}\left|u_{h}(t)\right|_{s}^{2} \mathrm{~d} t & \leq\left\|u_{h}(0)\right\|_{\Omega}^{2}+\left(\gamma^{-1}+T\right) \int_{0}^{\tau}\|F\|_{h}^{2} \mathrm{~d} t+\int_{0}^{\tau} T^{-1}\left\|u_{h}(t)\right\|_{\Omega}^{2} \mathrm{~d} t \\
& \leq C\left(\left\|u_{h}(0)\right\|_{\Omega}^{2}+\left(\gamma^{-1}+T\right) \int_{0}^{\tau}\|F\|_{h}^{2} \mathrm{~d} t\right)
\end{aligned}
$$

which concludes the proof.

For the material derivative we can prove the similar bound

Corollary 1 Let $u_{h}$ solve (2.13) with $\gamma>0$ then there holds

$\int_{0}^{T}\left\|h^{\frac{1}{2}} \mathcal{L} u_{h}\right\|_{\Omega}^{2} \mathrm{~d} t \leq C_{\beta} \zeta(\gamma)^{2}\left(\left\|u_{h}(0)\right\|_{\Omega}^{2}+\int_{0}^{T}\left(h\|F\|_{0}^{2}+\left(\beta_{\infty}+h\|\nabla \boldsymbol{\beta}\|_{\infty}^{2} T\right)\|F\|_{h}^{2}\right) \mathrm{d} t\right)$, where $\zeta(\gamma)=\gamma^{\frac{1}{2}}+\gamma^{-\frac{1}{2}}$.

\section{Proof}

$$
\int_{0}^{T}\left\|h^{\frac{1}{2}} \mathcal{L} u_{h}\right\|_{\Omega}^{2} \mathrm{~d} t=\int_{0}^{T}\left(\mathcal{L} u_{h}, h \pi_{h} \mathcal{L} u_{h}\right)_{\Omega} \mathrm{d} t+\int_{0}^{T}\left\|h^{\frac{1}{2}}\left(I-\pi_{h}\right) \mathcal{L} u_{h}\right\|_{\Omega}^{2} \mathrm{~d} t=T_{1}+T_{2} .
$$

To bound the term $T_{1}$ we use the formulation (2.13) to obtain

$$
\left(\mathcal{L} u_{h}, h \pi_{h} \mathcal{L} u_{h}\right)_{\Omega}=F\left(h \pi_{h} \mathcal{L} u_{h}\right)-\gamma s\left(u_{h}, h \pi_{h} \mathcal{L} u_{h}\right) .
$$

For the first term on the right hand side we see that using the first definition of (3.1) and the stability of the $L^{2}$-projection there holds

$$
F\left(h \pi_{h} \mathcal{L} u_{h}\right) \leq\|F\|_{0}\left\|h \pi_{h} \mathcal{L} u_{h}\right\|_{\Omega} \leq h^{\frac{1}{2}}\|F\|_{0}\left\|h^{\frac{1}{2}} \mathcal{L} u_{h}\right\|_{\Omega} .
$$

For the second term we use (2.9) and the $L^{2}$-stability of the projection to get

$$
\gamma s\left(u_{h}, h \pi_{h} \mathcal{L} u_{h}\right) \leq \gamma s\left(u_{h}, u_{h}\right)^{\frac{1}{2}} s\left(h \pi_{h} \mathcal{L} u_{h}, h \pi_{h} \mathcal{L} u_{h}\right)^{\frac{1}{2}} \leq C \gamma \beta_{\infty}^{\frac{1}{2}}\left|u_{h}\right|_{s}\left\|h^{\frac{1}{2}} \mathcal{L} u_{h}\right\|_{\Omega} .
$$

Observe that in the last inequality a factor $h^{\frac{1}{2}}$ is lost due to the application of (2.9). Collecting these bounds we see that

$$
T_{1} \leq \int_{0}^{T}\left(h\|F\|_{0}^{2}+C^{2} \gamma^{2} \beta_{\infty}\left|u_{h}\right|_{s}^{2}+\frac{1}{2}\left\|h^{\frac{1}{2}} \mathcal{L} u_{h}\right\|_{\Omega}^{2}\right) \mathrm{d} t
$$

To bound $T_{2}$ we note that by the definition of the $L^{2}$-projection $\left\|h^{\frac{1}{2}}\left(I-\pi_{h}\right) \mathcal{L} u_{h}\right\|_{\Omega} \leq$ $\left\|h^{\frac{1}{2}}\left(\mathcal{L} u_{h}-v_{h}\right)\right\|_{\Omega}$ for all $v_{h} \in V_{h}$ and apply (2.11) and the fact that $\partial_{t} u_{h} \in V_{h}$, leading to

$$
T_{2}=\int_{0}^{T} \inf _{v_{h} \in V_{h}}\left\|h^{\frac{1}{2}}\left(\boldsymbol{\beta} \cdot \nabla u_{h}-v_{h}\right)\right\|_{\Omega}^{2} \mathrm{~d} t \leq C_{s} \int_{0}^{T}\left(\beta_{\infty}\left|u_{h}\right|_{s}^{2}+h\|\nabla \boldsymbol{\beta}\|_{\infty}^{2}\left\|u_{h}\right\|_{\Omega}^{2}\right) \mathrm{d} t .
$$

The claim follows by the bounds on $T_{1}$ and $T_{2}$ and the result of Theorem 1 . 
Remark 2 Observe that the presence of both positive and negative powers of $\gamma$ in $\zeta$, shows that the estimate degenerates both for vanishing stabilization and for too strong stabilization. If $\gamma$ goes to infinity the solution has to become $C^{1}$ and the solution will in this case coincide with the standard Galerkin approximation in the $C^{1}$-subspace, which is unstable, see discussion in [18].

\section{Error Estimates for the Stabilized Formulation (2.13)}

Using the stability estimates of Theorem 1 it is straightforward to derive the error estimate (1.1) for smooth solutions. Below we will also use Corollary 1 to obtain an optimal order $O\left(h^{k}\right)$ error estimate for the material derivative.

Then we will assume that $f \in L^{2}(0, T ; \Omega)$ in (2.3) so that we only have $u \in$ $L^{2}(0, T ; \Omega)$. In this case we will show that the stabilized finite element method still converges in a weaker norm.

Theorem 2 Let $u_{0} \in H^{k+1}(\Omega), f \in L^{2}\left(0, T ; H^{k+1}(\Omega)\right)$, let $u$ be the solution of (2.1) and $u_{h}$ the solution of (2.13). Then there holds, for all $T>0$

$\left\|\left(u-u_{h}\right)(\cdot, T)\right\|_{\Omega}+\gamma\left(\int_{0}^{T}\left|u_{h}\right|_{s}^{2} \mathrm{~d} t\right)^{\frac{1}{2}} \leq C_{\beta} \zeta(\gamma) h^{k+\frac{1}{2}}\left(\|f\|_{L^{2}\left(0, T ; H^{k+1}(\Omega)\right)}+\left\|u_{0}\right\|_{H^{k+1}(\Omega)}\right)$

and

$$
\left(\int_{0}^{T}\left\|\mathcal{L}\left(u-u_{h}\right)\right\|_{\Omega}^{2} \mathrm{~d} t\right)^{\frac{1}{2}} \leq C_{\beta} \zeta(\gamma)^{2} h^{k}\|u\|_{H^{1}\left(0, T ; H^{k+1}(\Omega)\right)},
$$

where $\zeta(\gamma):=\gamma^{\frac{1}{2}}+\gamma^{-\frac{1}{2}}$ and $C_{\beta}$ depends on $\beta_{\infty}$ and $\|\nabla \boldsymbol{\beta}\|_{\infty}$ and $T$.

Proof This result is a consequence of the stability of Theorem 1, the consistency and (2.11). It is standard material (see [24, Section 76.4]) however for completeness we include the short proof.

Using standard approximation estimates there holds [10, Lemma 5.6]

$$
\left\|\beta_{\infty}^{\frac{1}{2}} h^{-\frac{1}{2}}\left(u-\pi_{h} u\right)\right\|_{\Omega}+\left|u-\pi_{h} u\right|_{s} \leq C \beta_{\infty}^{\frac{1}{2}} h^{k+\frac{1}{2}}|u|_{H^{k+1}(\Omega)} .
$$

Hence by applying a triangle inequality we only need to consider the discrete error $e_{h}=$ $\pi_{h} u-u_{h}$. Injecting it in (2.1) and using (2.13) we see that

$$
\left(\mathcal{L} e_{h}, v_{h}\right)+\gamma s\left(e_{h}, v_{h}\right)=F_{\pi}\left(v_{h}\right)
$$

with $F_{\pi}\left(v_{h}\right)=\left(\partial_{t}\left(\pi_{h} u-u\right), v_{h}\right)_{\Omega}+\left(\boldsymbol{\beta} \cdot \nabla\left(\pi_{h} u-u\right), v_{h}\right)_{\Omega}+\gamma s\left(\pi_{h} u, v_{h}\right)$. Applying Theorem 1 we see that

$$
\left\|e_{h}(T)\right\|_{\Omega}^{2}+\gamma \int_{0}^{T}\left|e_{h}\right|_{s}^{2} \mathrm{~d} t \leq C_{\beta} \int_{0}^{T}\left\|F_{\pi}\right\|_{h}^{2} \mathrm{~d} t+\left\|e_{h}(0)\right\|_{\Omega}^{2} .
$$

By the definition of $u_{h}(0), e_{h}(0)=0$. Since $\partial_{t} \pi_{h} u=\pi_{h} \partial_{t} u$ we have using $L^{2}$ orthogonality and intergration by parts

$$
F_{\pi}\left(v_{h}\right)=\left(u-\pi_{h} u, \boldsymbol{\beta} \cdot \nabla v_{h}-w_{h}\right)_{\Omega}+\gamma s\left(\pi_{h} u, v_{h}\right), \quad \forall w_{h} \in V_{h} .
$$


It now follows using the Cauchy-Schwarz inequality, (2.11) and (4.1) and recalling that under the regularity assumptions on data $u(t) \in H^{2}(\Omega)$, that

$$
\left\|F_{\pi}\right\|_{h} \leq C_{\beta} \zeta(\gamma) h^{k+\frac{1}{2}}|u|_{H^{k+1}(\Omega)} .
$$

The first claim then follows after an application of (2.3).

For the second inequality we apply Corollary 1 to see that, since $e_{h}(0)=0$,

$$
\int_{0}^{T}\left\|h^{\frac{1}{2}} \mathcal{L} e_{h}\right\|_{\Omega}^{2} \mathrm{~d} t \leq C \zeta(\gamma)^{2} \int_{0}^{T}\left(h\left\|F_{\pi}\right\|_{0}^{2}+\left(\beta_{\infty}+h\|\nabla \boldsymbol{\beta}\|_{\infty}^{2} T\right)\left\|F_{\pi}\right\|_{h}^{2}\right) \mathrm{d} t .
$$

It follows that we only need to bound $F$ in the stronger topology $\|\cdot\|_{0}$ to conclude. Using the Cauchy-Schwarz inequality and the inverse inequalities (2.6) and (2.9)

$$
\begin{aligned}
F_{\pi}\left(v_{h}\right) & =\left(u-\pi_{h} u, \boldsymbol{\beta} \cdot \nabla v_{h}\right)_{\Omega}+\gamma s\left(\pi_{h} u, v_{h}\right) \\
& \leq C \beta_{\infty}\left\|h^{-1}\left(u-\pi_{h} u\right)\right\|_{\Omega}\left\|v_{h}\right\|_{\Omega}+C \gamma h^{-\frac{1}{2}} \beta_{\infty}^{\frac{1}{2}}\left|\pi_{h} u\right|_{s}\left\|v_{h}\right\|_{\Omega} .
\end{aligned}
$$

It follows from (4.1) that

$$
\|F\|_{0} \leq C_{\beta}(1+\gamma) h^{k}|u|_{H^{k+1}(\Omega)} .
$$

Combining this bound for $\|F\|_{0}$ with the bound (4.2) in (4.3) we see that

$$
\int_{0}^{T}\left\|h^{\frac{1}{2}} \mathcal{L} e_{h}\right\|_{\Omega}^{2} \mathrm{~d} t \leq C_{\beta} \zeta(\gamma)^{4} h^{2 k+1} \int_{0}^{T}|u|_{H^{k+1}(\Omega)}^{2} \mathrm{~d} t
$$

and we conclude using the approximation bound

$$
\left\|\mathcal{L}\left(u-\pi_{h} u\right)\right\|_{\Omega} \leq C\left(h^{k+1}\left\|\partial_{t} u\right\|_{H^{k+1}(\Omega)}+\beta_{\infty} h^{k}\|u\|_{H^{k+1}(\Omega)}\right)
$$

and the triangle inequality.

Remark 3 Note that the error estimate on the material derivative is optimal compared with the approximation properties of the finite element space. In the corresponding analysis for (2.4) only $\|F\|_{0}$ may be used for the upper bound in Theorem 1, resulting in a bound that is suboptimal by $O\left(h^{\frac{1}{2}}\right)$.

\subsection{Rough Solutions: Convergence in Weak Norms}

Assume now that we have $f \in L^{2}((0, T) ; \Omega)$ in (2.13) and $u_{0} \in L^{2}(\Omega)$. Then $u \in$ $L^{2}((0, T) ; \Omega)$ is the best we can hope for, making the error estimates of Theorem 2 invalid. However if we estimate the error in a weaker norm, we can still obtain an error bound with convergence order, provided a stabilized method is used. For $\psi \in H_{p e r}^{1}(\Omega)$ consider the adjoint problem

$$
\begin{aligned}
-\mathcal{L} \varphi & =0, \\
\varphi(\cdot, T) & =\psi .
\end{aligned}
$$

This problem admits a unique solution and by (2.3)

$$
\sup _{t \in(0, T)}\|\varphi(t)\|_{H^{1}(\Omega)} \leq C_{\beta}\|\psi\|_{H^{1}(\Omega)} .
$$

Let $V:=H_{p e r}^{1}(\Omega)$ and introduce the dual norm

$$
\|v\|_{V^{\prime}}:=\sup _{w \in V \backslash 0} \frac{\langle v, w\rangle_{V^{\prime}, V}}{\|w\|_{V}},
$$


where $\langle v, w\rangle_{V^{\prime}, V}$ is a space duality pairing that we can identify with the $L^{2}$-scalar product for $v \in L^{2}(\Omega)$. We now proceed using duality to prove an a posteriori bound

Proposition 1 (A posteriori error bound) Let $u$ be the solution of (2.1) with $f \in$ $L^{2}(0, T ; \Omega)$ and $u_{0} \in L^{2}(\Omega)$ and $u_{h}$ the solution of (2.13), with $\gamma \geq 0$. Then there holds, for all $T>0$ and for all $\psi \in V$,

$$
\begin{aligned}
\frac{\left(\left(u-u_{h}\right)(\cdot, T), \psi\right)_{\Omega}}{\|\psi\|_{V}} \leq & C_{\beta} h\left\|u_{0}-\pi_{h} u_{0}\right\|_{\Omega} \\
& +C_{\beta} \int_{0}^{T}\left(\inf _{v_{h} \in V_{h}} h\left\|f-\boldsymbol{\beta} \cdot \nabla u_{h}-v_{h}\right\|_{\Omega}+\gamma h^{\frac{1}{2}}\left|u_{h}\right|_{s}\right) \mathrm{d} t .
\end{aligned}
$$

Proof Using the adjoint equation and integration by parts we see that for any $\psi \in H_{p e r}^{1}(\Omega)$,

$$
\begin{aligned}
\left(\left(u-u_{h}\right)(\cdot, T), \psi\right)_{\Omega}= & \left(\left(u-u_{h}\right)(\cdot, T), \psi\right)_{\Omega}+\int_{0}^{T}\left(u-u_{h},-\mathcal{L} \varphi\right)_{\Omega} \mathrm{d} t \\
= & \left(u_{0}-\pi_{h} u_{0}, \varphi(\cdot, 0)\right)_{\Omega}+\int_{0}^{T}\left(\mathcal{L}\left(u-u_{h}\right), \varphi\right)_{\Omega} \mathrm{d} t \\
= & \left(u_{0}-\pi_{h} u_{0},\left(I-\pi_{h}\right) \varphi(\cdot, 0)\right)_{\Omega} \\
& +\int_{0}^{T}\left(\left(\mathcal{L}\left(u-u_{h}\right), \varphi-\pi_{h} \varphi\right)_{\Omega}+\gamma s\left(u_{h}, \pi_{h} \varphi\right)\right) \mathrm{d} t .
\end{aligned}
$$

Considering the terms of the right hand side we see that

$$
\begin{aligned}
\left(u_{0}-\pi_{h} u_{0},\left(I-\pi_{h}\right) \varphi(\cdot, 0)\right)_{\Omega} & \leq C h\left\|u_{0}-\pi_{h} u_{0}\right\| \Omega\|\nabla \varphi(\cdot, 0)\| \Omega, \\
\left(\left(\mathcal{L}\left(u-u_{h}\right), \varphi-\pi_{h} \varphi\right)_{\Omega}\right. & \leq C h \inf _{v_{h} \in V_{h}}\left\|f-\mathcal{L} u_{h}-v_{h}\right\|_{\Omega}\|\nabla \varphi\|_{\Omega} \\
& =C h \inf _{v_{h} \in V_{h}}\left\|f-\boldsymbol{\beta} \cdot \nabla u_{h}-v_{h}\right\| \Omega\|\nabla \varphi\|_{\Omega}
\end{aligned}
$$

and

$$
s\left(u_{h}, \pi_{h} \varphi\right) \leq\left|u_{h}\right|_{s} h^{\frac{1}{2}} \beta_{\infty}^{\frac{1}{2}}\|\nabla \varphi\|_{\Omega} .
$$

It follows that

$$
\begin{aligned}
& \left(u_{0}-\pi_{h} u_{0},\left(I-\pi_{h}\right) \varphi(\cdot, 0)\right)_{\Omega}+\int_{0}^{T}\left(\left(\mathcal{L}\left(u-u_{h}\right), \varphi-\pi_{h} \varphi\right)_{\Omega}+\gamma s\left(u_{h}, \pi_{h} \varphi\right)\right) \mathrm{d} t \\
& \leq C\left(h\left\|u_{0}-\pi_{h} u_{0}\right\|+\int_{0}^{T}\left(\inf _{v_{h} \in V_{h}} h\left\|f-\boldsymbol{\beta} \cdot \nabla u_{h}-v_{h}\right\|_{\Omega}+\gamma \beta_{\infty}^{\frac{1}{2}} h^{\frac{1}{2}}\left|u_{h}\right|_{s}\right) \mathrm{d} t\right) \\
& \quad \times \sup _{t \in(0, T)}\|\varphi(t)\|_{H^{1}(\Omega)} .
\end{aligned}
$$

We end the proof by applying the stability (4.4).

Remark 4 A posteriori error estimates in negative norms for stationary first order pde was introduced in [30] and the case of transient problems using stabilized FEM in [9]. Observe that this a posteriori error estimate can not in general be sharp, indeed for a smooth solution, by Theorem 2 we get $O\left(h^{k+1}\right)$ convergence in the dual norm. This follows by observing that since we may take $v_{h}=\partial_{t} u_{h}$ and $f=\mathcal{L} u$,

$$
\inf _{v_{h} \in V_{h}} h\left\|f-\boldsymbol{\beta} \cdot \nabla u_{h}-v_{h}\right\|_{\Omega} \leq h\left\|\mathcal{L}\left(u-u_{h}\right)\right\|_{\Omega}
$$


and then applying the second bound of Theorem 2 . We see that compared to the $L^{2}$-estimate we have lost another power $h^{\frac{1}{2}}$. Sharp residual type a posteriori error estimates in the $L^{2}$ norm for transport equations in dimension $>1$, so far to the best of my knowledge, have only been obtained under a saturation assumption and using a stabilized finite element method, or a dG method with upwind flux [8].

Theorem 3 (A priori error estimate for rough solutions) Let $u$ be the solution of (2.1) with $f \in L^{2}\left(0, T ; L^{2}(\Omega)\right)$ and $u_{0} \in L^{2}(\Omega)$ and $u_{h}$ that of (2.13) with $\gamma>1$. Then there holds

$$
\sup _{t \in[0, T)}\left\|\left(u-u_{h}\right)(\cdot, t)\right\|_{V^{\prime}} \leq C_{\beta}(\zeta(\gamma)+1) h^{\frac{1}{2}}\left(\|f\|_{L^{2}\left(0, T ; L^{2}(\Omega)\right)}+\left\|u_{0}\right\|_{\Omega}\right),
$$

with $\zeta(\gamma)=\gamma^{\frac{1}{2}}+\gamma^{-\frac{1}{2}}$.

Proof By definition

$$
\left\|u-u_{h}\right\|_{V^{\prime}}=\sup _{w \in V \backslash 0} \frac{\left(u-u_{h}, w\right)_{\Omega}}{\|w\|_{V}} .
$$

Applying Proposition 1 we see that, after a Cauchy-Schwarz inequality in time, for any $T>0$,

$$
\begin{aligned}
\|\left(u-u_{h}(\cdot, T) \|_{V^{\prime}} \leq\right. & C_{\beta} h\left\|u_{0}-\pi_{h} u_{0}\right\|_{\Omega} \\
& +C_{\beta} h^{\frac{1}{2}} T^{\frac{1}{2}}\left(\int_{0}^{T}\left(\inf _{v_{h} \in V_{h}} h\left\|f-\boldsymbol{\beta} \cdot \nabla u_{h}-v_{h}\right\|_{\Omega}^{2}+\gamma^{2}\left|u_{h}\right|_{s}^{2}\right) \mathrm{d} t\right)^{\frac{1}{2}} .
\end{aligned}
$$

Then noting that by (2.11) there holds

$$
\inf _{v_{h} \in V_{h}} h\left\|f-\boldsymbol{\beta} \cdot \nabla u_{h}-v_{h}\right\|_{\Omega}^{2} \leq h\|f\|_{\Omega}^{2}+C_{s}\left(\left|u_{h}\right|_{s}^{2}+h\|\nabla \boldsymbol{\beta}\|_{\infty}^{2}\left\|u_{h}\right\|_{\Omega}^{2}\right)
$$

we see that all the a posteriori terms depending on $u_{h}$ are either on the form $\left|u_{h}\right|_{s}$ or on the form $\left\|u_{h}\right\|_{\Omega}^{2}$ and we conclude by applying Theorem 1 .

\subsection{Time Discretization and Stabilized Methods}

As a rule of thumb any time integrator with non-trivial imaginary stability boundary extending into the complex plane will be stable and accurate in the sense (1.1), possibly under a CFL condition depending on $\boldsymbol{\beta}$ and $\gamma$. In particular any time discretization method allowing for a time discrete version of an energy estimate of the type in Theorem 1 may be applied and will lead to optimal error estimates similar to those above. This includes all A-stable schemes, backward differentiation methods of first and second order, the Crank-Nicolson method. Explicit methods with good stability properties such as explicit strongly stable Runge-Kutta (RK) methods of order higher than, or equal to, 3 are stable $[12,40-43]$. Similar stability results are expected to hold for Adams-Bashforth (AB) methods of order 3, 4, 7, 8 under standard hyperbolic CFL, $\delta t \leq C o h$, where $\delta t$ denotes the timestep and $C o$ the Courant number. See for instance [31] for a discussion of time-discretization of advection-diffusion equation, [25] for a discussion of the stability boundaries of $\mathrm{AB}$ methods and [13] for numerical experiments using $\mathrm{AB} 3$. All these methods are energy stable regardless of whether or not stabilization is added. The second order RK method is energy stable under hyperbolic CFL only for piecewise affine approximation and with added stabilization of the form (2.8) [12] (for dG FEM and affine approximation 
upwind stabilization must be added [42]). In the general case (no stabilization, higher polynomial approximation) the RK2 method is stable only under a slightly more strict $C F L$ condition, indeed one needs to assume $d t \leq \mathrm{Coh}^{\frac{4}{3}}$, with $C o$ fixed, but small enough. This condition is the same for both $\mathrm{cG}$ and dG methods (see [12, 42]). Recently an analysis of the second order backward differentiation formula and the Crank-Nicolson method (AB2) with convection extrapolated to second order from previous time steps was proposed for the discretization of (2.13) [13]. It was shown that these schemes are stable under similar conditions as the RK2 scheme. Such multi step schemes are particularly appealing in the context of IMEX methods for convection-diffusion and hence provide a one-stage alternative to the RK2 IMEX method analysed in [11].

\section{Weighted Error Estimates}

In this section we will consider the slightly more technically advanced case of weighted estimates. The idea is to show that stabilization makes information follow the characteristics similarly as in the physics. This means that for solutions with a localized sharp layer, the dependence of a local error in the smooth zone on the regularity of the exact solution decreases exponentially with the distance to the singularity. Hence locally large gradients in the solution can not destroy the solution globally. This is not the case for approximations produced using cG FEM without stabilization. These results touch at the very essence of stabilized FEM, unfortunately their proofs are quite technical and therefore these results in my opinion have received less attention than they deserve. Here we try to give the simplest possible exposition of these ideas, without striving for optimality of exponential decay or generality of meshes. We let the domain be infinite $(L=\infty)$ and let $u_{0}$ have compact support. To simplify the discussion assume that $\beta \equiv e_{x}$, where $e_{x}$ is the Cartesian unit vector in the $x$-direction, so that $\boldsymbol{\beta} \cdot \nabla u=\partial_{x} u$. Since here $\beta_{\infty}=1$, below the dependence on the speed will not be tracked. First the case of a globally smooth solution will be considered (Theorem 4). The objective is to obtain an estimate for the error in some subdomain $\Omega_{0}(t) \subset \Omega$ defined as

$$
\Omega_{0}(t):=\left\{x \in \Omega:\left|x_{0}+\boldsymbol{\beta} t-\boldsymbol{x}\right|<r_{0}\right\}
$$

for some $\boldsymbol{x}_{0} \in \Omega$ and some $r_{0}>0$. The derivatives of $u$ are assumed to be moderate in a neighbourhood of $\Omega_{0}$ and we will prove that the accuracy in this subdomain is independent of large derivatives in other parts of the domain, provided they are sufficiently far away, relative to the mesh size. This is achieved using weights so that the effect of portions of the domain where locally the Sobolev norm is large decays exponentially with the distance to $\Omega_{0}$. Then we will show how the arguments of the smooth case can be used to prove accuracy in $\Omega_{0}$ in the case where the solution is locally only $L^{2}$ in the far field (Corollary 2). The key message is that the local accuracy of the approximation depends only on the local smoothness of the exact solution and that perturbations due to roughness in the solution is exponentially damped, except along characteristics. Finally we will discuss how the arguments can be extended to bounded domains with weakly imposed boundary condition and time discretization.

Let $\varphi \in C^{k+1}(\Omega)$ be a smooth positive function defined using polar/spherical coordinates, depending only on $r(x)=\left|x_{0}-\boldsymbol{x}\right|$, with $\varphi^{\prime}(r) \leq 0, \varphi(r)=1, r \leq r_{0}$, $\varphi(r) \sim \exp \left(-\left(r-r_{0}\right) / \sigma\right), r>r_{0}$, with $\sigma=K \sqrt{h}, K>1$, and for some $C>0$,

$$
\left|\partial_{r}^{l} \varphi(r)\right| \leq C \sigma^{-l} \varphi(r), \quad l \geq 1 .
$$




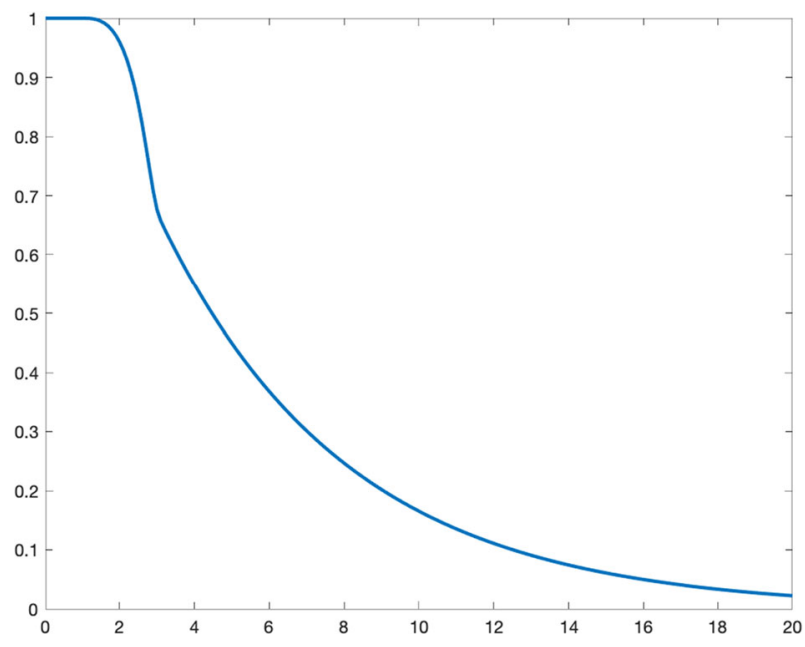

Fig. 1 Example of the radial cross section of $\varphi \in C^{1}(\Omega)$ with $r_{0}=1$ and $\sigma=5$

Remark 5 For the case $k=1$ we only require $\varphi \in C^{1}(\Omega)$. An example of such a function with $r_{0}=1$ and $\sigma=5$ is given in Fig. 1 for illustration.

Define $\varpi(\boldsymbol{x}, t)=\varphi(r(\boldsymbol{x}-\boldsymbol{\beta} t))$ then, since $\varpi$ follows the characteristics $\mathcal{L} \varpi=0$, and

$$
\left|D^{l} \varpi\right| \leq C \sigma^{-l} \varpi, \quad l \geq 1
$$

where the derivatives are taken with respect to space or time. The objective is to prove stability and error estimates in the weighted norm

$$
\|v\|_{\varpi}:=\|\varpi v\|_{\Omega} .
$$

The same notation will be used occasionally below with different weight functions. The rationale for the design of the weight function is that for all $v \in L^{\infty}\left(0, T ; L^{2}(\Omega)\right)$ with $\mathcal{L} v \in L^{2}(0, T ; \Omega)$, by partial integration in space and time,

$$
\int_{0}^{T}\left(\partial_{t} v, \varpi^{2} v\right)_{\Omega}=\|v(\cdot, T)\|_{\varpi}^{2}-\|v(\cdot, 0)\|_{\varpi}^{2}-\int_{0}^{T}\left(v, \partial_{t} \varpi^{2} v+\varpi^{2} \partial_{t} v\right)_{\Omega} \mathrm{d} t
$$

and

$$
\left(\boldsymbol{\beta} \cdot \nabla v, \varpi^{2} v\right)_{\Omega}=-\left(v,\left(\boldsymbol{\beta} \cdot \nabla \varpi^{2}\right) v+\varpi^{2} \boldsymbol{\beta} \cdot \nabla v\right)_{\Omega},
$$

there holds

$$
\begin{aligned}
\int_{0}^{T}\left(\mathcal{L} v, \varpi^{2} v\right)_{\Omega} \mathrm{d} t= & \|v(\cdot, T)\|_{\varpi}^{2}-\|v(\cdot, 0)\|_{\varpi}^{2} \\
& -\int_{0}^{T}(v, \underbrace{\left(\mathcal{L} \varpi^{2}\right)}_{=0} v)_{\Omega}+\left(v, \varpi^{2} \mathcal{L} v\right)_{\Omega} \mathrm{d} t .
\end{aligned}
$$

Hence

$$
\int_{0}^{T}\left(\mathcal{L} v, \varpi^{2} v\right)_{\Omega} \mathrm{d} t=\frac{1}{2}\|v(\cdot, T)\|_{\varpi}^{2}-\frac{1}{2}\|v(\cdot, 0)\|_{\varpi}^{2}
$$


and therefore the following stability is satisfied by the continuous equation, (2.1), $\forall \sigma>0$,

$$
\frac{1}{2}\|u(\cdot, T)\|_{\varpi}^{2} \leq \frac{1}{2}\|u(\cdot, 0)\|_{\varpi}^{2}+\int_{0}^{T}\|f\|_{\varpi}\|u\|_{\varpi} \mathrm{d} t
$$

from which we conclude

$$
\sup _{t \in(0, T)}\|u(\cdot, t)\|_{\varpi} \leq\|u(\cdot, 0)\|_{\varpi}+2 \int_{0}^{T}\|f\|_{\varpi} \mathrm{d} t .
$$

This relation expresses that the solution is transported along the characteristics. The influence across characteristics will be damped exponentially as $\exp (-d / \sigma)$. However in the continuous case, since the bound holds for all $\sigma>0$ the cut-off is sharp.

The aim is to make the error analysis for the solution of (2.13) reproduce this type of localization. For the purposes of analysis we introduce the weighted stabilization operator

$$
s_{\varpi}\left(v_{h}, w_{h}\right)=\sum_{F \in \mathcal{F}} \int_{F} h_{F}^{2} \varpi^{2} \llbracket \nabla v_{h} \rrbracket \llbracket \nabla w_{h} \rrbracket \mathrm{d} s \text {, with semi-norm }|w|_{s, \varpi}:=s_{\varpi}(w, w)^{\frac{1}{2}}
$$

and note that $s\left(v_{h}, \varpi^{2} w_{h}\right)=s_{\varpi}\left(v_{h}, w_{h}\right)$. Also recall the following weighted versions of (2.11) from [14, Lemma 3.1, equation (3.1) and (3.2)], here $\left.\boldsymbol{\beta}_{0}\right|_{S} \in \mathbb{R}^{n}$ is some piecewise constant per element,

$$
\left\|h^{\frac{1}{2}}\left(\boldsymbol{\beta}_{0} \cdot \nabla v_{h}-\pi_{h} \boldsymbol{\beta}_{0} \cdot \nabla v_{h}\right)\right\|_{\varpi}^{2} \leq C_{w s} \| \boldsymbol{\beta}_{0}\left|v_{h}\right|_{s, \varpi}^{2}
$$

and

$$
\left\|h^{\frac{1}{2}}\left(\boldsymbol{\beta} \cdot \nabla\left(\varpi^{2} v_{h}\right)-\pi_{h}\left(\boldsymbol{\beta} \cdot \nabla\left(\varpi^{2} v_{h}\right)\right)\right)\right\|_{\varpi^{-1}}^{2} \leq C_{w s}\left|v_{h}\right|_{s, \varpi}^{2}+C_{\beta} K^{-2}\left\|v_{h}\right\|_{\varpi}^{2} .
$$

The second bound differs from the bound in [14], since there the derivative of $v_{h}$ appears in the second term of the right hand side. The proof however is similar. For completeness we detail it in Appendix. We will need to use approximation in the weighted norm and therefore collect some results on the $L^{2}$-projection in the following lemmas. The first one is taken from [2] and we refer to this reference for the proof. The following two are variations on results from [14] and for completeness we give the proofs in Appendix. We note that all the above inequalities hold both for the weight $\varpi$ and $\varpi^{-1}$, since by the construction of the weight,

$$
\left|\nabla \varpi^{-1}\right|=\left|\varpi^{-2} \nabla \varpi\right| \leq C \varpi^{-2} \sigma^{-1} \varpi=C \sigma^{-1} \varpi^{-1} .
$$

It follows that (5.1) is satisfied also for $\varpi^{-1}$.

Lemma 1 (Stability $L^{2}$-projection) Let $\pi_{h}$ denote the $L^{2}$-projection onto $V_{h}$. Then, if $\phi$ is a function satisfying

$$
|\nabla \phi(x)| \leq v h^{-1}|\phi(x)|,
$$

for some $v>0$, sufficiently small then there holds

$$
\begin{aligned}
\left\|\pi_{h} v\right\|_{\phi} & \leq C\|v\|_{\phi}, \\
\left\|\nabla \pi_{h} v\right\|_{\phi} & \leq C\|\nabla v\|_{\phi}
\end{aligned}
$$

and

$$
\left\|\nabla \pi_{h} v\right\|_{\phi} \leq C h^{-1}\|v\|_{\phi}, \quad \forall v \in H^{1}(\Omega) .
$$

Proof The estimates (5.5)-(5.7) are taken verbatim from [2, bounds (1.7)-(1.9)] (see also [22, Appendix]). 
The above stability estimates allows us to prove bounds on the $L^{2}$-error in the weighted norm.

Lemma 2 (Weighted approximation) Let $\pi_{h}$ denote the $L^{2}$-projection onto $V_{h}$. Then for $h^{\frac{1}{2}} / K$ sufficiently small and $I_{\delta}=[t-\delta t, t+\delta t] \cap[0, T]$ with $\delta t \in \mathbb{R}^{+}, \delta t \sim h$, there holds

$$
\begin{gathered}
\max _{(x, t) \in S \times I_{\delta}} \varpi(x, t)\|v\|_{S} \leq 2 \min _{t \in I_{\delta}}\|v \varpi(\cdot, t)\|_{S}, \quad \forall v \in L^{2}(S), \\
\left\|\left(v-\pi_{h} v\right)\right\|_{\varpi}+h\left\|\nabla\left(v-\pi_{h} v\right)\right\|_{\varpi} \leq C h^{k+1}\left\|D^{k+1} v\right\|_{\varpi}, \quad \forall v \in H^{k+1}(\Omega)
\end{gathered}
$$

and

$$
\left|v-\pi_{h} v\right|_{s, \varpi} \leq C h^{k+\frac{1}{2}}\left\|D^{k+1} v\right\|_{\varpi}, \quad \forall v \in H^{k+1}(\Omega) .
$$

For the analysis we also need the following interpolation estimates on weighted discrete functions.

Lemma 3 (Super approximation) Let $v_{h} \in V_{h}$. Assume that $h^{\frac{1}{2}} / K$ is sufficiently small. Then there holds

$$
\left\|\varpi^{2} v_{h}-\pi_{h}\left(\varpi^{2} v_{h}\right)\right\|_{\varpi^{-1}}+h\left\|\nabla\left(\varpi^{2} v_{h}-\pi_{h}\left(\varpi^{2} v_{h}\right)\right)\right\|_{\varpi^{-1}} \leq C h^{\frac{1}{2}} K^{-1}\left\|v_{h}\right\|_{\varpi}
$$

and

$$
\left(\sum_{S \in \mathcal{T}}\left\|\varpi^{-1} \nabla\left(\varpi^{2} v_{h}-\pi_{h}\left(\varpi^{2} v_{h}\right)\right)\right\|_{\partial S}^{2}\right)^{\frac{1}{2}} \leq C h^{-1} K^{-1}\left\|v_{h}\right\|_{\varpi}
$$

We will now derive a weighted stability estimate for the finite element formulation (2.13). First use similar arguments as for (5.2) to obtain for any $v_{h} \in C^{1}\left(0, T ; V_{h}\right)$,

$$
\int_{0}^{T}\left(\mathcal{L} v_{h}, \varpi^{2} v_{h}\right)_{\Omega} \mathrm{d} t=\frac{1}{2}\left\|v_{h}(\cdot, T)\right\|_{\varpi}^{2}-\frac{1}{2}\left\|v_{h}(\cdot, 0)\right\|_{\varpi}^{2}
$$

and, since $\varpi \in C^{1}(\Omega)$ we see that

$$
s\left(v_{h}, \varpi^{2} v_{h}\right)=\left|v_{h}\right|_{s, \varpi}^{2} .
$$

Therefore,

$$
\left\|v_{h}(\cdot, T)\right\|_{\varpi}^{2}+2 \gamma \int_{0}^{T}\left|v_{h}\right|_{s, \varpi}^{2} \mathrm{~d} t=2 \int_{0}^{T}\left(\left(\mathcal{L} v_{h}, \varpi^{2} v_{h}\right)_{\Omega}+\gamma s\left(v_{h}, \varpi^{2} v_{h}\right)\right) \mathrm{d} t+\left\|v_{h}(\cdot, 0)\right\|_{\varpi}^{2} .
$$

However, since $\varpi^{2} v_{h} \notin V_{h}$ the equality can not be used directly for the finite element formulation. We need to show that stability similar to (5.13) can be obtained by testing by some interpolant of $\varpi^{2} v_{h}$.

Proposition 2 (Weighted stability) Let $\gamma>0, K>1$. Assume that $h^{\frac{1}{2}} / K$ is sufficiently small. For all $v_{h} \in C^{1}\left(0, T ; V_{h}\right)$ there holds

$$
\begin{aligned}
\left\|v_{h}(\cdot, T)\right\|_{\varpi}^{2}+\gamma \int_{0}^{T}\left|v_{h}\right|_{s, \varpi}^{2} \mathrm{~d} t \leq & C / K^{2} \int_{0}^{T}\left\|v_{h}\right\|_{\varpi}^{2} \mathrm{~d} t \\
& +2 \int_{0}^{T}\left(\left(\mathcal{L} v_{h}, w_{h}\right)_{\Omega}+\gamma s\left(v_{h}, w_{h}\right)\right) \mathrm{d} t+\left\|v_{h}(\cdot, 0)\right\|_{\varpi}^{2},
\end{aligned}
$$

where $w_{h}=\pi_{h} \varpi^{2} v_{h}$ and the constant $C \sim \gamma+\gamma^{-1}$. 
Proof Starting from the equality (5.13) we add and subtract the finite element formulation tested with some function $w_{h}$,

$$
\begin{aligned}
\left\|v_{h}(\cdot, T)\right\|_{\varpi}^{2}+2 \gamma \int_{0}^{T}\left|v_{h}\right|_{s, \varpi}^{2} \mathrm{~d} t= & 2 \int_{0}^{T}\left(\left(\mathcal{L} v_{h}, \varpi^{2} v_{h}-w_{h}\right)_{\Omega}+\gamma s\left(v_{h}, \varpi^{2} v_{h}-w_{h}\right)\right) \mathrm{d} t \\
& +2 \int_{0}^{T}\left(\left(\mathcal{L} v_{h}, w_{h}\right)_{\Omega}+\gamma s\left(v_{h}, w_{h}\right)\right) \mathrm{d} t+\left\|v_{h}(\cdot, 0)\right\|_{\varpi}^{2} .
\end{aligned}
$$

We choose $w_{h}=\pi_{h}\left(\varpi^{2} v_{h}\right)$ to obtain, for an arbitrary $y_{h} \in V_{h}$

$$
\begin{aligned}
\left(\mathcal{L} v_{h}, \varpi^{2} v_{h}-\pi_{h}\left(\varpi^{2} v_{h}\right)\right)_{\Omega} & =\left(\boldsymbol{\beta} \cdot \nabla v_{h}-y_{h}, \varpi^{2} v_{h}-\pi_{h}\left(\varpi^{2} v_{h}\right)\right)_{\Omega} \\
& \leq \inf _{y_{h} \in V_{h}}\left\|h^{\frac{1}{2}}\left(\boldsymbol{\beta} \cdot \nabla v_{h}-y_{h}\right)\right\|_{\varpi} h^{-\frac{1}{2}}\left\|\left(\varpi^{2} v_{h}-\pi_{h}\left(\varpi^{2} v_{h}\right)\right)\right\|_{\varpi^{-1}} .
\end{aligned}
$$

Considering the stabilization term we see that

$$
s\left(v_{h}, \varpi^{2} v_{h}-\pi_{h}\left(\varpi^{2} v_{h}\right)\right) \leq\left|v_{h}\right|_{s, \varpi} h \beta_{\infty}^{\frac{1}{2}}\left(\sum_{F \in \mathcal{F}}\left\|\varpi^{-1} \llbracket \nabla\left(\varpi^{2} v_{h}-\pi_{h}\left(\varpi^{2} v_{h}\right)\right) \rrbracket\right\|_{F}^{2}\right)^{\frac{1}{2}} .
$$

Using the arithmetic-geometric inequality $a b \leq(2 \epsilon)^{-1} a^{2}+\left(\epsilon 2^{-1}\right) b^{2}$, to split the terms in the right hand side, with $\epsilon=2$ in (5.14), we obtain

$$
\begin{aligned}
& \left\|v_{h}(\cdot, T)\right\|_{\varpi}^{2}+\frac{7}{4} \gamma \int_{0}^{T}\left|v_{h}\right|_{s, \varpi}^{2} \mathrm{~d} t \leq \epsilon^{-1} \gamma^{-1} h^{-1} \int_{0}^{T} \underbrace{\left\|\left(\varpi^{2} v_{h}-\pi_{h}\left(\varpi^{2} v_{h}\right)\right)\right\|_{\varpi^{-1}}^{2}}_{T_{1}} \mathrm{~d} t \\
& +\gamma h^{2} \beta_{\infty} \int_{0}^{T} \underbrace{\sum_{F \in \mathcal{F}}\left\|\varpi^{-1} \llbracket \nabla\left(\varpi^{2} v_{h}-\pi_{h}\left(\varpi^{2} v_{h}\right)\right) \rrbracket\right\|_{F}^{2}}_{T_{2}} \mathrm{~d} t \\
& +\epsilon \gamma \int_{0}^{T} \underbrace{\inf _{y_{h} \in V_{h}}\left\|h^{\frac{1}{2}}\left(\boldsymbol{\beta} \cdot \nabla v_{h}-y_{h}\right)\right\|_{\varpi}^{2}}_{T_{3}} \mathrm{~d} t \\
& +2 \int_{0}^{T}\left(\left(\mathcal{L} v_{h}, w_{h}\right)_{\Omega}+\gamma s\left(v_{h}, w_{h}\right)\right) \mathrm{d} t+\left\|v_{h}(\cdot, 0)\right\|_{\varpi}^{2} .
\end{aligned}
$$

We need to bound the contributions $T_{1}, T_{2}$ and $T_{3}$ in terms of the quantities of the left hand side and $\left\|v_{h}\right\|_{\varpi}$. Using (5.11) immediately yields

$$
T_{1}=\left\|\left(\varpi^{2} v_{h}-\pi_{h}\left(\varpi^{2} v_{h}\right)\right)\right\|_{\varpi^{-1}}^{2} \leq C K^{-2} h\left\|v_{h}\right\|_{\varpi}^{2} .
$$

By distribution of the integrals over the faces on simplices, splitting the jumps on the contributions from the two sides and applying (5.12) there holds

$$
T_{2} \leq C \sum_{S \in \mathcal{T}}\left\|\varpi^{-1} \nabla\left(\varpi^{2} v_{h}-\pi_{h}\left(\varpi^{2} v_{h}\right)\right)\right\|_{\partial S}^{2} \leq C / K^{2} h^{-2}\left\|v_{h}\right\|_{\varpi}^{2} .
$$

Finally for the term $T_{3}$ apply the weighted stabilization bound (5.3), with $\boldsymbol{\beta}_{0} \equiv e_{x}$, where $e_{x}$ is the Cartesian unit vector in the $x$-direction

$$
T_{3}=\inf _{y_{h} \in V_{h}}\left\|h^{\frac{1}{2}}\left(\boldsymbol{\beta} \cdot \nabla v_{h}-y_{h}\right)\right\|_{\varpi}^{2} \leq C_{w s}\left|v_{h}\right|_{s, \varpi}^{2} .
$$


Collecting the bounds for $T_{1}-T_{3}$ and choosing $\epsilon=\left(2 C_{w s}\right)^{-1}$ we see that

$$
\begin{aligned}
\left\|v_{h}(\cdot, T)\right\|_{\varpi}^{2}+\gamma \int_{0}^{T}\left|v_{h}\right|_{s, \varpi}^{2} \mathrm{~d} t \leq & \left(\gamma^{-1}+\gamma\right) C / K^{2} \int_{0}^{T}\left\|v_{h}\right\|_{\varpi}^{2} \mathrm{~d} t \\
& +2 \int_{0}^{T}\left(\left(\mathcal{L} v_{h}, w_{h}\right)_{\Omega}+\gamma s\left(v_{h}, w_{h}\right)\right) \mathrm{d} t+\left\|v_{h}(\cdot, 0)\right\|_{\varpi}^{2} .
\end{aligned}
$$

Theorem 4 Assume that the hypothesis of Proposition 2 are satisfied. Let $u \in L^{\infty}(0, T$; $H^{k+1}(\Omega)$ ) be the solution of (2.1) and $u_{h}$ the solution of (2.13). Then for all $T>0$ there holds

$$
\left\|\left(u-u_{h}\right)(\cdot, T)\right\|_{\varpi} \leq C_{K} h^{k+\frac{1}{2}}\left(h\left\|D^{k+1} u(\cdot, T)\right\|_{\varpi}^{2}+\left(\gamma+\gamma^{-1}\right) \int_{0}^{T}\left\|D^{k+1} u\right\|_{\varpi}^{2} \mathrm{~d} t\right)^{\frac{1}{2}} .
$$

The constant $C_{K}$ grows exponentially in time with coefficient proportional to $\left(\gamma+\gamma^{-1}\right) K^{-2}$.

First note that we may split the error as $u-u_{h}=\underbrace{u-\pi_{h} u}_{=-\eta}+\underbrace{\pi_{h} u-u_{h}}_{=e_{h}}$ and by (5.9),

$$
\left\|\left(u-\pi_{h} u\right)(\cdot, T)\right\|_{\varpi} \leq C h^{k+1}\left\|D^{k+1} u(\cdot, T)\right\|_{\varpi} .
$$

By the triangle inequality we only need to prove the bound on $\left\|e_{h}(\cdot, T)\right\|_{\varpi}$.

Using the stability of Proposition 2 we see that, since $e_{h}(\cdot, 0)=0$,

$$
\begin{aligned}
\left\|e_{h}(\cdot, T)\right\|_{\varpi}^{2}+\gamma \int_{0}^{T}\left|e_{h}\right|_{s, \varpi}^{2} \mathrm{~d} t \leq & C / K^{2} \int_{0}^{T}\left\|e_{h}\right\|_{\varpi}^{2} \mathrm{~d} t \\
& +2 \int_{0}^{T}\left(\left(\mathcal{L} e_{h}, w_{h}\right)_{\Omega}+\gamma s\left(e_{h}, w_{h}\right)\right) \mathrm{d} t
\end{aligned}
$$

with $w_{h}=\pi_{h}\left(\varpi^{2} e_{h}\right)$. Now observe that the following consistency property holds

$$
\int_{0}^{T}\left(\mathcal{L}\left(e_{h}-\eta\right), v_{h}\right)_{\Omega}-\gamma s\left(u_{h}, v_{h}\right) \mathrm{d} t=0, \quad \forall v_{h} \in V_{h}
$$

and hence

$$
\int_{0}^{T}\left(\left(\mathcal{L} e_{h}, w_{h}\right)_{\Omega}+\gamma s\left(e_{h}, w_{h}\right)\right) \mathrm{d} t=\int_{0}^{T}\left(\left(\mathcal{L} \eta, w_{h}\right)_{\Omega}+\gamma s\left(\pi_{h} u_{h}, w_{h}\right)\right) \mathrm{d} t .
$$

This leads to a perturbation equation on the form

$$
\begin{aligned}
\left\|e_{h}(\cdot, T)\right\|_{\varpi}^{2}+\gamma \int_{0}^{T}\left|e_{h}\right|_{s, \varpi}^{2} \mathrm{~d} t \leq & C K^{-2} \int_{0}^{T}\left\|e_{h}\right\|_{\varpi}^{2} \mathrm{~d} t \\
& +2 \int_{0}^{T}\left(\left(\mathcal{L} \eta, w_{h}\right)_{\Omega}+\gamma s\left(\pi_{h} u_{h}, w_{h}\right)\right) \mathrm{d} t .
\end{aligned}
$$

Considering the first term of the second integral in the right hand side we have using that time derivation and the $L^{2}$-projection commute and the $L^{2}$-orthogonality of $\eta$

$$
\begin{aligned}
\left(\mathcal{L} \eta, w_{h}\right)_{\Omega} & =-\left(\eta, \boldsymbol{\beta} \cdot \nabla w_{h}-y_{h}\right)_{\Omega} \leq h^{-\frac{1}{2}}\|\eta\|_{\varpi} h^{\frac{1}{2}} \inf _{y_{h} \in V_{h}}\left\|\boldsymbol{\beta} \cdot \nabla w_{h}-y_{h}\right\|_{\varpi^{-1}} \\
& \leq h^{-1} \gamma^{-1} C\|\eta\|_{\varpi}^{2}+\frac{1}{4} \gamma\left|e_{h}\right|_{s, \varpi}^{2}+C \gamma / K^{2}\left\|e_{h}\right\|_{\varpi}^{2} .
\end{aligned}
$$


Here we used the inequality $a b \leq 4^{-1} a^{2}+b^{2}$ and that by the triangle inequality followed by the bounds (5.11), and (5.4) there holds

$$
\begin{aligned}
h^{\frac{1}{2}} \inf _{y_{h} \in V_{h}}\left\|\boldsymbol{\beta} \cdot \nabla w_{h}-y_{h}\right\|_{\varpi^{-1}} \leq & h^{\frac{1}{2}}\left\|\boldsymbol{\beta} \cdot \nabla \pi_{h}\left(\varpi^{2} e_{h}\right)-\boldsymbol{\beta} \cdot \nabla\left(\varpi^{2} e_{h}\right)\right\|_{\varpi^{-1}} \\
& +h^{\frac{1}{2}} \inf _{y_{h} \in V_{h}}\left\|\boldsymbol{\beta} \cdot \nabla\left(\varpi^{2} e_{h}\right)-y_{h}\right\|_{\varpi^{-1}} \\
\leq & h^{\frac{1}{2}} \beta_{\infty}\left\|\nabla\left(\pi_{h}\left(\varpi^{2} e_{h}\right)-\varpi^{2} e_{h}\right)\right\|_{\varpi^{-1}} \\
& +\left(C_{w s}\left|e_{h}\right|_{s, \varpi}^{2}+C_{\beta} K^{-2}\left\|e_{h}\right\|_{\varpi}^{2}\right)^{\frac{1}{2}} \\
\leq & C K^{-1}\left\|e_{h}\right\|_{\varpi}+C_{w s}\left|e_{h}\right|_{s, \varpi} .
\end{aligned}
$$

For the last term in the right hand side of (5.15) we have

$$
\begin{aligned}
s\left(\pi_{h} u_{h}, w_{h}\right)= & s\left(\pi_{h} u_{h}, \pi_{h}\left(\varpi^{2} e_{h}\right)-\varpi^{2} e_{h}\right)+s\left(\pi_{h} u_{h}, \varpi^{2} e_{h}\right) \\
\leq & C\left|\pi_{h} u_{h}\right|_{s, \varpi}^{2}+\frac{1}{4}\left|e_{h}\right|_{s, \varpi}^{2} \\
& +h^{2} \beta_{\infty}^{2} \sum_{F \in \mathcal{F}}\left\|\varpi^{-1} \llbracket \nabla\left(\varpi^{2} e_{h}-\pi_{h}\left(\varpi^{2} e_{h}\right)\right) \rrbracket\right\|_{F}^{2} .
\end{aligned}
$$

Applying the bound (5.12) to each term of the jump separately in the last term in the right hand side and collecting the estimates it follows that

$$
\left(\mathcal{L} \eta, w_{h}\right)_{\Omega}+\gamma s\left(\pi_{h} u_{h}, w_{h}\right) \leq C\left(\gamma\left|\pi_{h} u_{h}\right|_{s, \varpi}^{2}+h^{-1} \gamma^{-1}\|\eta\|_{\varpi}^{2}\right)+\frac{1}{2} \gamma\left|e_{h}\right|_{s, \varpi}^{2}+\gamma C / K^{2}\left\|e_{h}\right\|_{\varpi}^{2} .
$$

Applying this bound in (5.15) we have

$$
\begin{aligned}
\left\|e_{h}(\cdot, T)\right\|_{\varpi}^{2}+\frac{1}{2} \gamma \int_{0}^{T}\left|e_{h}\right|_{s, \varpi}^{2} \mathrm{~d} t \leq & C\left(\gamma+\gamma^{-1}\right) / K^{2} \int_{0}^{T}\left\|e_{h}\right\|_{\varpi}^{2} \mathrm{~d} t \\
& +C \int_{0}^{T}\left(\gamma\left|\pi_{h} u_{h}\right|_{s, \varpi}^{2}+h^{-1} \gamma^{-1}\|\eta\|_{\varpi}^{2}\right) \mathrm{d} t .
\end{aligned}
$$

Since the solution is assumed regular, $u(\cdot, t) \in H^{\frac{3}{2}+\epsilon}(\Omega), \epsilon>0$ we have $\left|\pi_{h} u_{h}\right|_{s, \varpi}^{2}=$ $|\eta|_{s, \varpi}^{2}$. Applying Lemma 2 yields

$$
\int_{0}^{T}\left(\gamma|\eta|_{s, \varpi}^{2}+h^{-1} \gamma^{-1}\|\eta\|_{\varpi}^{2}\right) \mathrm{d} t \leq C h^{2 k+1}\left(\gamma+\gamma^{-1}\right) \int_{0}^{T}\left\|D^{k+1} u\right\|_{\varpi}^{2} \mathrm{~d} t .
$$

The claim now follows by an application of Gronwall's inequality.

\subsection{Discussion of Estimates for Rough Solutions}

Consider the following subsets of $\Omega, \Omega_{0}(t):=\{x \in \Omega: \varpi(x, t)=1\}$ and $\Omega_{p}(t):=\{x \in$ $\left.\Omega: \varpi(x, t) \leq h^{p}, p>0\right\}$. Then denoting $d=\operatorname{dist}\left(\Omega_{0}, \Omega_{p}\right)$ it follows by the construction of $\varpi$ that

$$
d \sim K p \sqrt{h}|\log (h)|,
$$

and the following bound holds

$$
\left\|\left(u-u_{h}\right)(\cdot, T)\right\|_{\Omega_{0}} \leq C h^{k+\frac{1}{2}}\left(\max _{t \in[0, T]}\left\|D^{k+1} u\right\|_{L^{2}\left(\Omega \backslash \Omega_{p}\right)}+h^{p} \max _{t \in[0, T]}\left\|D^{k+1} u\right\|_{L^{2}\left(\Omega_{p}\right)}\right) .
$$


It follows that $D^{k+1} u$ can be large, $O\left(h^{-p}\right)$, in $\Omega_{p}$ without destroying the solution in $\Omega_{0}$. To apply the argument to $u_{0}$ that is only piecewise in $H^{k+1}$ one can use the weighted $L^{2}$ stability in the error analysis above and still obtain estimates. We present a sketch of this result in a corollary.

Corollary 2 Assume that the hypothesis of Proposition 2 are satisfied. Let $p=k+1$. Assume that $u \in L^{\infty}\left(0, T ; L^{2}(\Omega)\right)$, with $\left.u\right|_{\Omega \backslash \Omega_{p}} \in H^{k+1}\left(\Omega \backslash \Omega_{p}\right)$, for all $t \in[0, T]$ is the solution of (2.1) and $u_{h}$ the solution of (2.13). Then there holds (omitting for simplicity the dependence on $\gamma$ ).

$$
\left\|\left(u-u_{h}\right)(\cdot, T)\right\|_{\Omega_{0}} \leq C_{K} h^{k+\frac{1}{2}}\left(\max _{t \in[0, T]}\|u\|_{H^{k+1}\left(\Omega \backslash \Omega_{p}\right)}+\max _{t \in[0, T]}\|u\|_{L^{2}\left(\Omega_{p}\right)}\right) .
$$

Proof The proof follows that of Theorem 4 closely. We only need to substitute the $L^{2}$ projection for an interpolant with more local properties before applying approximation. Let the domain $\Omega_{p, i h}(t)$ be defined by the union of all the elements that intersect $\Omega_{p}(T)$ and an integer $i$ layers of nearest neighbours. The norm over $\Omega_{p, i h}(t)$ will be denoted $\|\cdot\|_{\Omega_{p, i h}}$. Let $C_{h}$ denote the Clément interpolant defined using local projections. It is well known [23, Lemma 1.127] that if for a given $S \in \mathcal{T}, \Delta_{S}$ denotes the set of simplices sharing at least one vertex with $S$ and for a face $F, \Delta_{F}$ denotes the set of simplices sharing at least one vertex with $F$, then

$$
\begin{aligned}
& \left\|v-C_{h} v\right\|_{H^{m}(S)} \leq C h^{l-m}\|v\|_{H^{l}\left(\Delta_{S}\right)}, \quad\left\|v-C_{h} v\right\|_{H^{m}(F)} \leq C h^{l-m-\frac{1}{2}}\|v\|_{H^{l}\left(\Delta_{F}\right)}, \\
& 0 \leq m \leq l \leq k+1 .
\end{aligned}
$$

It is then straightforward to use the approximation properties of $C_{h}$ in $\Omega \backslash \Omega_{p, 1 h}$ and the local stability of $C_{h}$ in $\Omega_{p, 1 h}$ to show the estimates

$$
\begin{aligned}
\left\|\left(u-C_{h} u\right)(\cdot, t)\right\|_{\varpi} & \leq C\left(h^{k+1}\left\|D^{k+1} u(\cdot, t)\right\|_{\Omega \backslash \Omega_{p}}+h^{p}\|u(\cdot, t)\|_{\Omega_{p, 2 h}}\right) \\
& \leq C h^{k+1}\left(\|u(\cdot, t)\|_{H^{k+1}\left(\Omega \backslash \Omega_{p}\right)}+\|u(\cdot, t)\|_{\Omega_{p}}\right)
\end{aligned}
$$

and

$$
\begin{aligned}
\left|C_{h} u(\cdot, t)\right|_{s, \varpi} & \leq C\left(h^{k+\frac{1}{2}}\left\|D^{k+1} u(\cdot, t)\right\|_{\Omega \backslash \Omega_{p}}+h^{-\frac{1}{2}+p}\|u(\cdot, t)\|_{\Omega_{p, 2 h}}\right) \\
& \leq C h^{k+\frac{1}{2}}\left(\|u(\cdot, t)\|_{H^{k+1}\left(\Omega \backslash \Omega_{p}\right)}+\|u(\cdot, t)\|_{\Omega_{p}}\right) .
\end{aligned}
$$

For the second inequality we divide $\left|C_{h} u(\cdot, t)\right|_{s, \varpi}$ into the sum over faces in $\Omega \backslash \Omega_{p, 1 h}$ and $\Omega_{p, 1 h}$. The two different sets are treated differently. For faces in $\Omega \backslash \Omega_{p, 1 h}$ we proceeded as

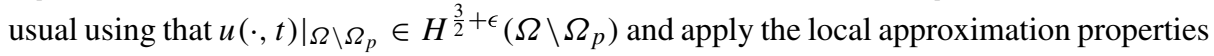
on faces of $C_{h}$ (second inequality of (5.17)). For faces in $\Omega_{p, 1 h}$ we can not use approximation and instead apply (2.10) and (2.6). We also used that $\left.\varpi\right|_{\Omega_{p, 1 h}} \leq C h^{p}$ by construction. Observe that by the weighted $L^{2}$-stability (5.5) we have

$\left\|\left(u-\pi_{h} u\right)(\cdot, T)\right\|_{\varpi} \leq\left\|\pi_{h}\left(u-C_{h} u\right)(\cdot, T)\right\|_{\varpi}+\left\|\left(u-C_{h} u\right)(\cdot, T)\right\|_{\varpi} \leq C\left\|\left(u-C_{h} u\right)(\cdot, T)\right\|_{\varpi}$

and hence as before we only need to prove the bound for $\left\|e_{h}(\cdot, T)\right\|_{\varpi}$. The inequality (5.16) still holds. To conclude we observe that using (5.20)

$$
\int_{0}^{T} h^{-1}\|\eta\|_{\varpi}^{2} \mathrm{~d} t \leq C \int_{0}^{T} h^{-1}\left\|u-C_{h} u\right\|_{\varpi}^{2} \mathrm{~d} t .
$$


By combining the inequality

$$
\left|v_{h}\right|_{s, \varpi} \leq C h^{-\frac{1}{2}}\left\|v_{h}\right\|_{\varpi}
$$

(that is immediate by (2.10), (2.6) and (5.8)) with (5.20) we also have

$$
\begin{aligned}
\int_{0}^{T}\left|\pi_{h} u\right|_{s, \varpi}^{2} \mathrm{~d} t & \leq 2 \int_{0}^{T}\left(\left|\pi_{h} u-C_{h} u\right|_{s, \varpi}^{2}+\left|C_{h} u_{h}\right|_{s, \varpi}^{2}\right) \mathrm{d} t \\
& \leq C \int_{0}^{T}\left(h^{-1}\left\|u-C_{h} u\right\|_{\varpi}^{2}+\left|C_{h} u_{h}\right|_{s, \varpi}^{2}\right) \mathrm{d} t .
\end{aligned}
$$

We conclude as before after applying (5.18) and (5.19) in (5.21) and (5.22).

\subsection{Time Discretization and Weakly Imposed Boundary Conditions}

In practice and in the numerical section below of course we need to include boundary conditions and time discretizations in the above arguments. Depending on the time-discretization this can be a challenging exercise, but we will here focus on the $\theta$-scheme and the main steps of its analysis using the ideas above in the case of the backward Euler scheme $(\theta=1)$. Boundary conditions are imposed weakly using the standard upwind technique known from discontinuous Galerkin methods. We consider a polygonal domain $\Omega$ and denote its boundary by $\Gamma:=\partial \Omega$ with outward pointing normal $n$. We decompose $\Gamma$ into an inflow part

$$
\Gamma_{-}:=\{x \in \Gamma: \boldsymbol{\beta}(x) \cdot n<0\}
$$

and an outflow part $\Gamma_{+}:=\partial \Omega \backslash \Gamma_{-}$. The space $V_{h}$ will here denote the standard finite element space of continuous piecewise polynomial functions, without boundary conditions defined on $\mathcal{T}$. We are now interested in the the solution of (2.1) with the additional inflow boundary condition

$$
u=g \text { on } \Gamma_{-},
$$

where $g \in L^{2}\left(0, T ; L_{\beta \cdot n}^{2}\left(\Gamma_{-}\right)\right)$with $L_{\boldsymbol{\beta} \cdot n}^{2}\left(\Gamma_{-}\right):=\left\{v: \Gamma_{-} \mapsto \mathbb{R}:\left\||\boldsymbol{\beta} \cdot n|^{\frac{1}{2}} v\right\|_{L^{2}\left(\Gamma_{-}\right)}<\infty\right\}$. We will assume that the $g, \Gamma_{-}$and $\Gamma_{+}$are such that the exact solution is smooth enough for our purposes. The timestep $\delta t:=T / N$ for some $N \in \mathbb{N}^{+}$will be assumed to satisfy $\delta t \leq C h$ for some $C>0$, and the discrete solution $u_{h}:=\left\{u_{h}^{n}\right\}_{n=0}^{N}$ collects the finite element approximations on the discrete time levels $t^{n}=n \delta t$. The so-called $\theta$-scheme takes the form: find $u_{h}^{n} \in V_{h}$ such that for $n=1,2,3 \ldots N$,

$\left(\mathcal{L}_{\theta}^{n} u_{h}, v_{h}\right)_{\Omega}+\left\langle|\boldsymbol{\beta} \cdot n| u_{h}^{n_{\theta}}, v_{h}\right\rangle_{\Gamma_{-}}+s\left(u_{h}^{n_{\theta}}, v_{h}\right)=\left(f^{n_{\theta}}, v_{h}\right)_{\Omega}+\left\langle|\boldsymbol{\beta} \cdot n| g^{n_{\theta}}, v_{h}\right\rangle_{\Gamma_{-}}, \forall v_{h} \in V_{h}$,

where $u_{h}^{n_{\theta}}:=\theta u_{h}^{n}+(1-\theta) u_{h}^{n-1}, g^{n_{\theta}}:=g\left(\cdot, t^{n}+\theta \delta t\right), f^{n_{\theta}}:=f\left(\cdot, t^{n}+\theta \delta t\right)$,

$$
\mathcal{L}_{\theta}^{n} u_{h}:=\delta t^{-1}\left(u_{h}^{n}-u_{h}^{n-1}\right)+\boldsymbol{\beta} \cdot \nabla u_{h}^{n_{\theta}}, \quad \theta \in[1 / 2,1]
$$

and $u_{h}^{0}=\pi_{h} u_{0}$. Compared to the time continuous analysis we have two additional points to study

1. the time discrete character of the equation,

2. the boundary penalty term. 
We recall that the theta scheme includes the well-known backward Euler scheme $(\theta=1)$ and the Crank-Nicolson scheme $(\theta=1 / 2)$. A complete analysis of the $\theta$ scheme is beyond the scope of the present paper. To give some insight in the validity of the above arguments in the fully discrete case we will show the modifications necessary to prove Proposition 2 in the time discrete case with weakly imposed boundary conditions, for $\theta=1$. Theorem 4 then follows using the arguments above and standard truncation error analysis. We will then show numerically that also the Crank-Nicolson scheme enjoys the local accuracy property. For further evidence of the local accuracy property we refer to [12, Section 5.2 and Fig. 1] for examples using explicit Runge-Kutta methods and [13, Section 6] for examples using explicit extrapolated multistep methods. For the analysis we need the following Lemma the proof of which is given in the Appendix.

Lemma 4 Let $\varpi_{n}(x)=\varpi\left(x, t_{n}\right)$, where $\varpi$ is a weightfunction satisfying (5.1) and $v_{h} \in$ $V_{h}$, then for $\delta t$ small enough there holds

$$
\left\|v_{h} \int_{t_{n-1}}^{t_{n}} \partial_{t} \varpi \mathrm{d} t\right\|_{\Omega}+\left\|v_{h}\left|\int_{t_{n-1}}^{t_{n}} \int_{t}^{t_{n}} \partial_{t}^{2} \varpi^{2} \mathrm{~d} s \mathrm{~d} t\right|^{\frac{1}{2}}\right\|_{\Omega} \leq C K^{-1} \delta t^{\frac{1}{2}}\left\|v_{h}\right\|_{\varpi_{n}} .
$$

The following weighted $L^{2}$-stability estimate is the key ingredient of the analysis of the fully discrete scheme.

Proposition 3 Consider the scheme (5.23) with $\theta=1$, then assuming $\delta t<1$ small enough there holds, with $w_{h}^{n}=\pi_{h} \varpi^{2} v_{h}^{n}$,

$$
\begin{aligned}
& \left\|v_{h}^{N}\right\|_{\varpi_{N}}^{2}+\sum_{n=1}^{N}\left\|v_{h}^{n}-v_{h}^{n-1}\right\|_{\varpi_{n}}^{2}+\delta t \sum_{n=1}^{N}\left(\left\||\boldsymbol{\beta} \cdot n|^{\frac{1}{2}} v_{h}^{n} \varpi_{n}\right\|_{\Gamma}^{2}+\gamma\left|v_{h}^{n}\right|_{s, \varpi_{n}}^{2}\right) \\
& \quad \leq C_{K}\left(\left\|v_{h}^{0}\right\|_{\varpi_{0}}^{2}+\delta t \sum_{n=1}^{N}\left(\left(\mathcal{L}_{\theta}^{n} v_{h}, w_{h}^{n}\right)_{\Omega}+\left\langle|\boldsymbol{\beta} \cdot n| v_{h}^{n}, w_{h}^{n}\right\rangle_{\Gamma_{-}}+\gamma s\left(v_{h}^{n}, w_{h}^{n}\right)\right)\right) .
\end{aligned}
$$

The constant $C_{K}$ grows exponentially in time with exponential coefficient $1 / K^{2}$.

Proof First we observe that using standard partial integration and $\nabla \cdot \boldsymbol{\beta}=0$ we have

$$
\begin{aligned}
& \left(\boldsymbol{\beta} \cdot \nabla v_{h}, \varpi^{2} v_{h}\right)_{\Omega}+\left\langle|\boldsymbol{\beta} \cdot n| v_{h}, \varpi^{2} v_{h}\right\rangle_{\Gamma_{-}} \\
& =-\left(\boldsymbol{\beta} \cdot \nabla v_{h}, \varpi^{2} v_{h}\right)_{\Omega}-\left(v_{h},\left(\boldsymbol{\beta} \cdot \nabla \varpi^{2}\right) v_{h}\right)_{\Omega}+\left\langle|\boldsymbol{\beta} \cdot n| v_{h}, \varpi^{2} v_{h}\right\rangle_{\Gamma_{+}} .
\end{aligned}
$$

As a consequence

$\left(\boldsymbol{\beta} \cdot \nabla v_{h}, \varpi^{2} v_{h}\right)_{\Omega}+\left\langle|\boldsymbol{\beta} \cdot n| v_{h}, \varpi^{2} v_{h}\right\rangle_{\Gamma_{-}}=-\frac{1}{2}\left(v_{h},\left(\boldsymbol{\beta} \cdot \nabla \varpi^{2}\right) v_{h}\right)_{\Omega}+\frac{1}{2}\left\langle|\boldsymbol{\beta} \cdot n| v_{h}, \varpi^{2} v_{h}\right\rangle_{\Gamma}$.

We also have

$$
\left(v_{h}^{n}-v_{h}^{n-1}, \varpi_{n}^{2} v_{h}^{n}\right)_{\Omega}=\frac{1}{2}\left\|v_{h}^{n}\right\|_{\varpi_{n}}^{2}+\frac{1}{2}\left\|v_{h}^{n}-v_{h}^{n-1}\right\|_{\varpi_{n}}^{2}-\frac{1}{2}\left\|v_{h}^{n-1}\right\|_{\varpi_{n}}^{2} .
$$


It follows that

$$
\begin{aligned}
\delta t & \sum_{n=1}^{N}\left(\left(\mathcal{L}_{\theta}^{n} v_{h}, \varpi_{n}^{2} v_{h}^{n}\right)_{\Omega}+\left\langle|\boldsymbol{\beta} \cdot n| v_{h}^{n}, \varpi_{n}^{2} v_{h}^{n}\right\rangle_{\Gamma_{-}}+\gamma s\left(v_{h}^{n}, \varpi_{n}^{2} v_{h}^{n}\right)\right) \\
= & \frac{1}{2}\left\|v_{h}^{N}\right\|_{\varpi_{N}}^{2}+\frac{1}{2} \sum_{n=1}^{N}\left(\left\|v_{h}^{n}-v_{h}^{n-1}\right\|_{\varpi_{n}}^{2}-\left(\left(v_{h}^{n-1}\right)^{2}, \varpi_{n}^{2}-\varpi_{n-1}^{2}\right)_{\Omega}\right)-\frac{1}{2}\left\|v_{h}^{0}\right\|_{\varpi_{0}}^{2} \\
& \quad-\frac{1}{2} \delta t \sum_{n=1}^{N}\left(\left(v_{h}^{n}\right)^{2}, \boldsymbol{\beta} \cdot \nabla \varpi_{n}^{2}\right)_{\Omega}+\frac{1}{2} \delta t \sum_{n=1}^{N}\left(\left\||\boldsymbol{\beta} \cdot n|^{\frac{1}{2}} v_{h}^{n} \varpi_{n}\right\|_{\Gamma}^{2}+2 \gamma s\left(v_{h}^{n}, \varpi_{n}^{2} v_{h}^{n}\right)\right) .
\end{aligned}
$$

Identifying the terms in the right hand side that do not have a sign we see that we need to control

$$
\left.\sum_{n=1}^{N}\left(\left(v_{h}^{n-1}\right)^{2}, \varpi_{n}^{2}-\varpi_{n-1}^{2}\right)_{\Omega}+\delta t\left(\left(v_{h}^{n}\right)^{2}, \boldsymbol{\beta} \cdot \nabla \varpi_{n}^{2}\right)_{\Omega}\right) .
$$

We rewrite the first term

$$
\left(\left(v_{h}^{n-1}\right)^{2},\left(\varpi_{n}^{2}-\varpi_{n-1}^{2}\right)\right)_{\Omega}=\left(\left(v_{h}^{n-1}\right)^{2}-\left(v_{h}^{n}\right)^{2}, \varpi_{n}^{2}-\varpi_{n-1}^{2}\right)_{\Omega}+\left(\left(v_{h}^{n}\right)^{2},\left(\varpi_{n}^{2}-\varpi_{n-1}^{2}\right)\right)_{\Omega} .
$$

For the first term on the right hand side we develop $a^{2}-b^{2}=(a+b)(a-b)$ and apply Cauchy-Schwarz inequality and the arithmetic-geometric inequality, followed by Lemma 4 and the inequality (5.8) to obtain the bound

$$
\begin{aligned}
& \left(\left(v_{h}^{n-1}\right)^{2}-\left(v_{h}^{n}\right)^{2}, \varpi_{n}^{2}-\varpi_{n-1}^{2}\right)_{\Omega}=\left(\left(v_{h}^{n-1}+v_{h}^{n}\right)\left(v_{h}^{n-1}-v_{h}^{n}\right), \varpi_{n}^{2}-\varpi_{n-1}^{2}\right)_{\Omega} \\
& \quad=\left(\left(v_{h}^{n-1}+v_{h}^{n}\right)\left(v_{h}^{n-1}-v_{h}^{n}\right),\left(\varpi_{n}+\varpi_{n-1}\right) \int_{t_{n-1}}^{t_{n}} \partial_{t} \varpi(\cdot, t) \mathrm{d} t\right)_{\Omega} \\
& \quad \geq-\epsilon^{-1}\left\|\left(v_{h}^{n}+v_{h}^{n-1}\right) \int_{t_{n-1}}^{t_{n}} \partial_{t} \varpi(\cdot, t) \mathrm{d} t\right\|_{\Omega}^{2}-\frac{\epsilon}{2}\left(\left(v_{h}^{n}-v_{h}^{n-1}\right)^{2}, \varpi_{n}^{2}+\varpi_{n-1}^{2}\right)_{\Omega} \\
& \quad \geq-C K^{-2} \epsilon^{-1} \delta t\left(\left\|v_{h}^{n}\right\|_{\varpi_{n}}^{2}+\left\|v_{h}^{n-1}\right\|_{\varpi_{n-1}}^{2}\right)-\frac{C \epsilon}{2}\left\|v_{h}^{n}-v_{h}^{n-1}\right\|_{\varpi_{n}}^{2} .
\end{aligned}
$$

Considering the remaining terms, using the relation $\mathcal{L} \varpi^{2}=0$, and applying once again Lemma 4, yields the bound

$$
\begin{aligned}
\left(\left(v_{h}^{n}\right)^{2}, \varpi_{n}^{2}-\varpi_{n-1}^{2}\right)_{\Omega}+\delta t\left(\left(v_{h}^{n}\right)^{2}, \boldsymbol{\beta} \cdot \nabla \varpi_{n}^{2}\right)_{\Omega} & =\left(\left(v_{h}^{n}\right)^{2}, \int_{t_{n-1}}^{t_{n}} \partial_{t} \varpi^{2} \mathrm{~d} t-\delta t \partial_{t} \varpi_{n}^{2}\right)_{\Omega} \\
& =\left(\left(v_{h}^{n}\right)^{2}, \int_{t_{n-1}}^{t_{n}} \int_{t_{n}}^{t} \partial_{t t} \varpi^{2} \mathrm{~d} s \mathrm{~d} t\right)_{\Omega} \\
& \geq-\delta t C / K^{2}\left\|v_{h}^{n}\right\|_{\varpi_{n}}^{2} .
\end{aligned}
$$

Taking $\epsilon$ sufficiently small so that $C \epsilon / 2 \leq 1 / 4$ it follows that

$$
\begin{aligned}
& \left\|v_{h}^{N}\right\|_{\varpi_{N}}^{2}+\sum_{n=1}^{N}\left(\left\|v_{h}^{n}-v_{h}^{n-1}\right\|_{\varpi_{n}}^{2}+\delta t \sum_{n=1}^{N}\left(\left\||\boldsymbol{\beta} \cdot n|^{\frac{1}{2}} v_{h}^{n} \varpi_{n}\right\|_{\Gamma}^{2}+\gamma\left|v_{h}^{n}\right|_{s, \varpi_{n}}^{2}\right)\right. \\
& \leq C\left(\left\|v_{h}^{0}\right\|_{\varpi_{0}}^{2}+\delta t \sum_{n=1}^{N}\left(\left(\mathcal{L}_{\theta}^{n} v_{h}, \varpi_{n}^{2} v_{h}^{n}\right)_{\Omega}+\left\langle|\boldsymbol{\beta} \cdot n| v_{h}^{n}, \varpi_{n}^{2} v_{h}^{n}\right\rangle_{\Gamma_{-}}\right.\right. \\
& \left.\left.\quad+\gamma s\left(v_{h}^{n}, \varpi_{n}^{2} v_{h}^{n}\right)+C K^{-2}\left\|v_{h}^{n}\right\|_{\varpi_{n}}^{2}\right)\right) .
\end{aligned}
$$


Proceeding as before we add and subtract $w_{h}^{n}:=\pi_{h}\left(\varpi_{n}^{2} v_{h}^{n}\right)$ in the right slot of the bilinear forms of the right hand side

$$
\begin{aligned}
& \left\|v_{h}^{N}\right\|_{\varpi_{N}}^{2}+\sum_{n=1}^{N}\left\|v_{h}^{n}-v_{h}^{n-1}\right\|_{\varpi_{n}}^{2}+\delta t \sum_{n=1}^{N}\left(\left\||\boldsymbol{\beta} \cdot n|^{\frac{1}{2}} v_{h}^{n} \varpi_{n}\right\|_{\Gamma}^{2}+\gamma\left|v_{h}^{n}\right|_{s, \varpi_{n}}^{2}\right) \\
& \leq C\left(\left\|v_{h}^{0}\right\|_{\varpi_{0}}^{2}+\delta t \sum_{n=1}^{N}\left(\left(\mathcal{L}_{\theta}^{n} v_{h}, w_{h}\right)_{\Omega}+\left\langle|\boldsymbol{\beta} \cdot n| v_{h}^{n}, w_{h}\right\rangle_{\Gamma_{-}}+s\left(v_{h}^{n}, w_{h}\right)+\delta t C\left\|v_{h}^{n}\right\|_{\varpi_{n}}^{2}\right)\right. \\
& \left.\quad+\delta t \sum_{n=1}^{N}\left(\left(\mathcal{L}_{\theta}^{n} v_{h}, \varpi_{n}^{2} v_{h}^{n}-w_{h}\right)_{\Omega}+\left\langle|\boldsymbol{\beta} \cdot n| v_{h}^{n}, \varpi_{n}^{2} v_{h}^{n}-w_{h}\right\rangle_{\Gamma_{-}}+\gamma s\left(v_{h}^{n}, \varpi_{n}^{2} v_{h}^{n}-w_{h}\right)\right)\right) .
\end{aligned}
$$

Only the term introduced for the weak imposition of boundary conditions differs from the time-continuous analysis. For this term we observe that

$$
\left\langle|\boldsymbol{\beta} \cdot n| v_{h}^{n}, \varpi_{n}^{2} v_{h}^{n}-w_{h}\right\rangle_{\Gamma_{-}} \geq-\epsilon\left\||\boldsymbol{\beta} \cdot n|^{\frac{1}{2}} v_{h}^{n} \varpi_{n}\right\|_{\Gamma}^{2}-\frac{\beta_{\infty}}{4 \epsilon}\left\|\varpi_{n}^{-1}\left(\varpi_{n}^{2} v_{h}^{n}-\pi_{h} \varpi_{n}^{2} v_{h}^{n}\right)\right\|_{\Gamma_{-}}^{2} .
$$

For the second term on the right hand side we have the bound

$$
\left\|\varpi_{n}^{-1}\left(\varpi_{n}^{2} v_{h}^{n}-\pi_{h} \varpi_{n}^{2} v_{h}^{n}\right)\right\|_{\Gamma_{-}}^{2} \leq C / K^{2}\left\|v_{h}^{n}\right\|_{\varpi}^{2} .
$$

This follows by applying the trace inequality (2.10), the properties of $\varpi$ and the inequality (5.11). Proceeding as in the time-continuous case we then obtain the bound

$$
\begin{aligned}
& \left\|v_{h}^{N}\right\|_{\varpi_{N}}^{2}+\sum_{n=1}^{N}\left\|v_{h}^{n}-v_{h}^{n-1}\right\|_{\varpi_{n}}^{2}+\delta t \sum_{n=1}^{N}\left(\left\||\boldsymbol{\beta} \cdot n|^{\frac{1}{2}} v_{h}^{n} \varpi_{n}\right\|_{\Gamma}^{2}+\gamma\left|v_{h}^{n}\right|_{s, \varpi_{n}}^{2}\right) \\
& \leq C\left(\left\|v_{h}^{0}\right\|_{\varpi_{0}}^{2}+\delta t \sum_{n=1}^{N}\left(\left(\mathcal{L}_{\theta}^{n} v_{h}, w_{h}\right)_{\Omega}+\left\langle|\boldsymbol{\beta} \cdot n| v_{h}^{n}, w_{h}\right\rangle_{\Gamma_{-}}+\gamma s\left(v_{h}^{n}, w_{h}\right)+K^{-2}\left\|v_{h}^{n}\right\|_{\varpi_{n}}^{2}\right)\right) .
\end{aligned}
$$

Choosing $\delta t$ sufficiently small the term $\delta t C K^{-2}\left\|v_{h}^{N}\right\|_{\varpi_{N}}^{2}$ in the right hand side can be absorbed in the left hand side and we conclude by an application of the discrete Gronwall's inequality.

Remark 6 A consequence of the previous analysis is that the proposed method can be used in the context of problems, where the boundary or initial data is unknown or partially known. Assume for example that $g$ is unknown and replaced by zero. Then, since the effect of the erroneous boundary condition is damped exponentially for non-characteristic directions, the solution can still be approximated with good accuracy in subsets $\Omega_{0}$ whose domain of dependence is sufficiently far from the boundary. Similarly if the initial data is unknown in some parts of the domain, the solution will still remain accurate in subdomains where the initial data in the domain of dependence is known. This result is a time-dependent analogue to the analysis of [16].

\section{Numerical Examples}

All numerical examples were produced using the package FreeFEM++ [29]. The method (5.23) is considered with $\theta=1 / 2$, corresponding to the second order Crank-Nicolson scheme. This choice was made to minimize the perturbation of the global energy estimate by the time-discretization. The consistent mass matrix is used and exact quadrature is applied to 
all the forms. We first consider transport in the disc $\Omega:=\left\{(x, y) \in \mathbb{R}^{2}: x^{2}+y^{2}<1\right\}$ under the velocity field $\boldsymbol{\beta}=(y,-x)$. Approximations are computed on a series of unstructured meshes. We set $f=0$ and consider two different functions $u_{0}$ as initial data. One is smooth

$$
u_{0}=e^{-30\left((x-0.5)^{2}+y^{2}\right)}
$$

and one is rough

$$
\tilde{u}_{0}=\left\{\begin{array}{lc}
1, & \sqrt{(x+0.5)^{2}+y^{2}}<0.2, \\
0 & \text { otherwise. }
\end{array}\right.
$$

The velocity field simply turns the disc with the initial data and one full turn is computed so that the final solution should be equal to the initial data. Two numerical experiments are considered where the solution is approximated for the initial data $u_{0}$ and $u_{0}+\tilde{u}_{0}$.

We report the global error in the material derivative over the space time domain, the global $L^{2}$-norm of the error at the final time, and in the case where both the rough and the smooth initial data are combined, the error obtained in the smooth part, i.e. the $L^{2}$-norm over $\{(x, y) \in \Omega: x>0\}$. The discretization parameters for piecewise affine ( $P_{1}$ below) approximation have been chosen as $d t=\frac{1}{2} h=\pi /$ nele, where nele is the number of cell faces on the disc perimeter. For piecewise quadratic ( $P_{2}$ below) approximation $h=2 \pi /$ nele and $d t=\frac{1}{2} h^{\frac{3}{2}}$, to make the error of the time and space discretization similar. In the left panel of Fig. 2 the smooth and rough initial data, interpolated on a very fine mesh, are presented. In the middle panel the solution after one turn without stabilization and in the right panel the solution after one turn with stabilization for $P_{1}$, on the mesh resolution nele $=80$ are reported. We see that the sharp layers are smeared on this coarse mesh when the stabilized method is used, but contrary to the unstabilized case the smooth part of the solution is accurately captured.

In Fig. 3 the convergence of stabilized and unstabilized methods with $P_{1}$ and $P_{2}$ elements are compared for the smooth initial data. We observe that when the solution is globally smooth both methods perform well in the $L^{2}$-norm. Nevertheless, the improvement of the convergence rate for the stabilized method is clearly visible for both approximation spaces, both in the $L^{2}$-error and in the material derivative. The results when part of the solution is rough (initial data from Fig. 2, left plot) are reported in Fig. 4. Note that both methods have similar global error in the $L^{2}$-norm. The stabilized method on the other hand still has optimal convergence in the part where the solution is smooth, in accordance with the theory of Section 5. Its material derivative is also more stable under refinement. The unstabilized method has equally poor convergence in the smooth and in the rough part of the solution.

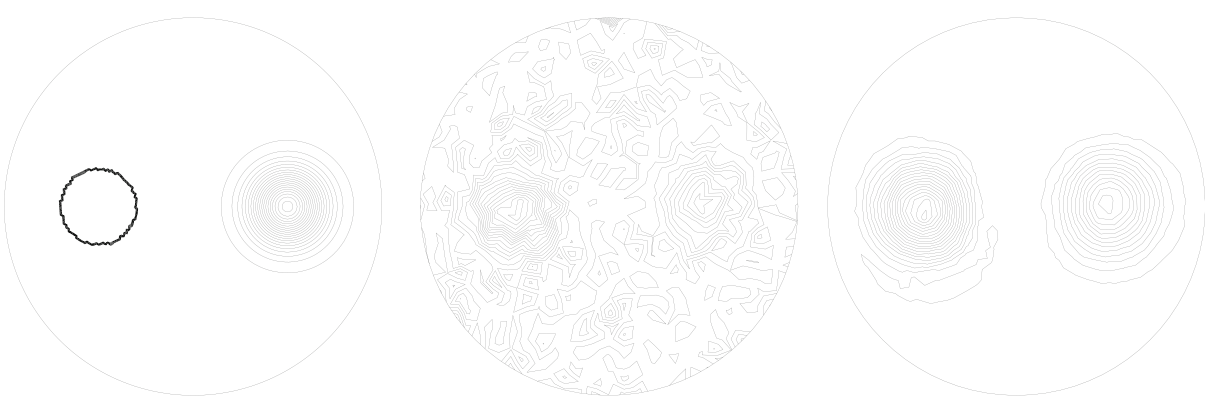

Fig. 2 From left to right: rough initial data on fine mesh $u_{0}+\tilde{u}_{0}$, unstabilized solution, stabilized solution (nele $=80$, one turn) 

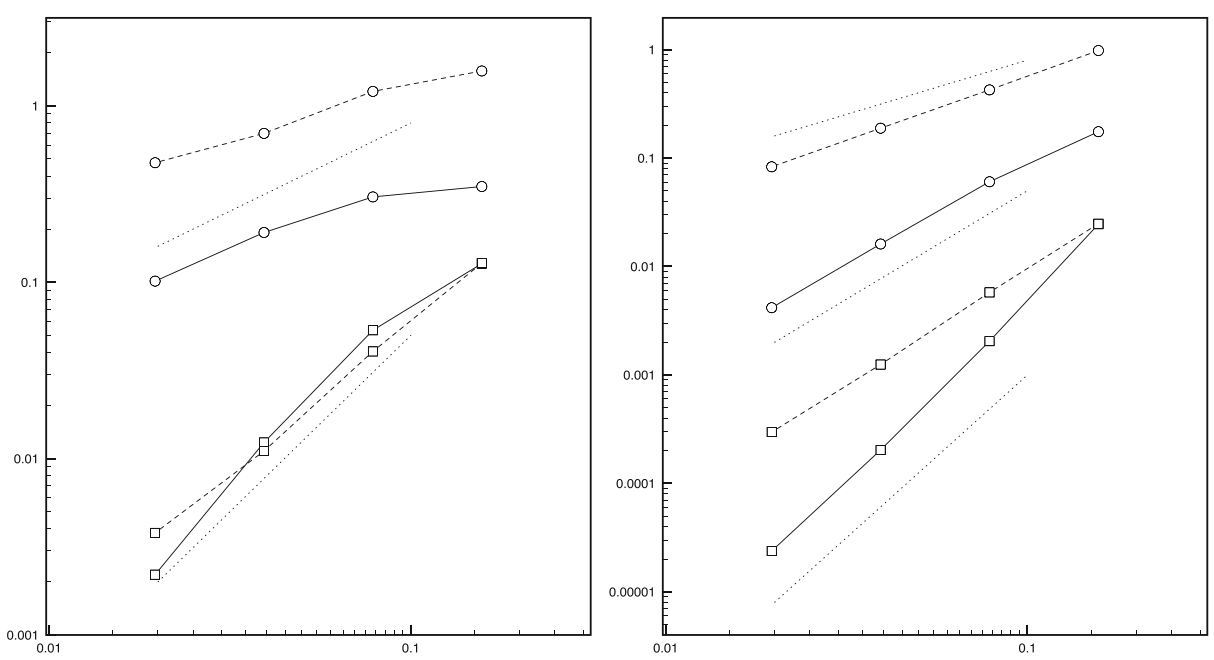

Fig. 3 Comparison of errors plotted against mesh size $h$ for stabilized (full line) and unstabilized (dashed line) methods with $P_{1}$ (left) and $P_{2}$ (right) approximation. Globally smooth initial data (6.1). The space time error in material derivative has circle markers. The final time global $L^{2}$-error has square markers. The dotted reference lines have slope 1,2 from top to bottom in the left graphic and 1,2,3 from top to bottom in the right graphic

\subsection{An Example with Inflow and Outflow and Weakly Imposed Boundary Conditions}

Here we consider transport in the unit square with $\beta=(1,0)^{T}$. We use a structured mesh with nele cell faces on the side of the square. The initial data consists of a cylinder of
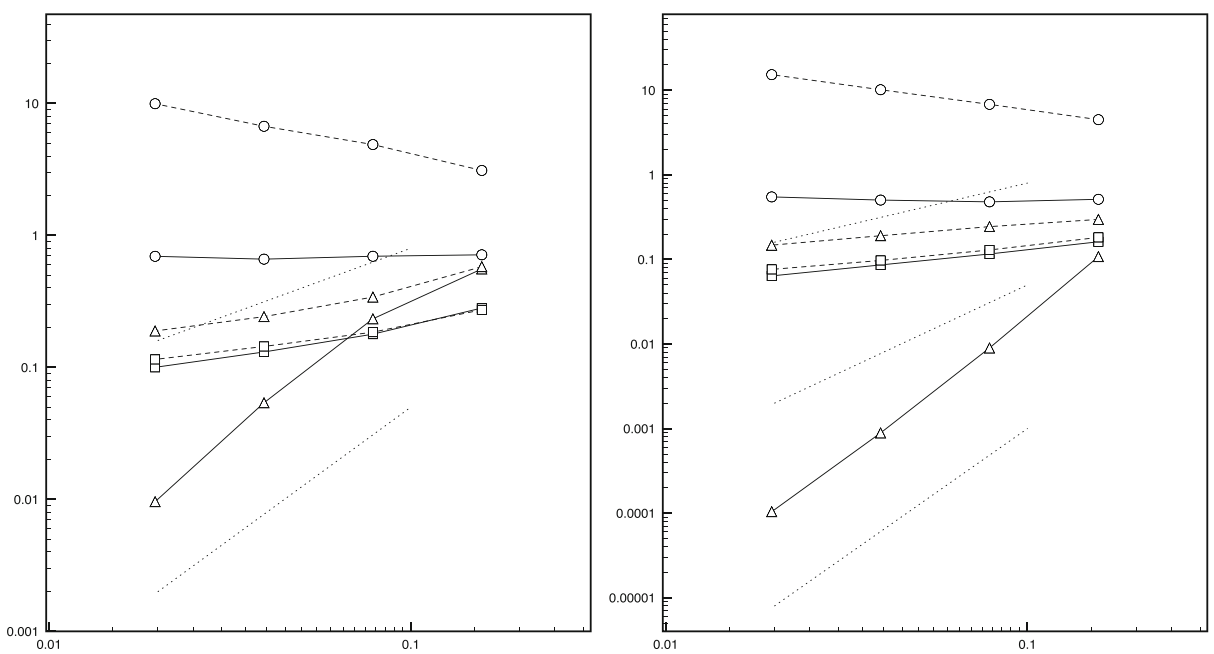

Fig. 4 Comparison of errors plotted against mesh size $h$ for stabilized (full line) and unstabilized (dashed line) methods with $P_{1}$ (left) and $P_{2}$ (right). Initial data from Fig. 2 (left plot). The space time error in material derivative has circle markers. The final time global $L^{2}$-error has square markers and the final time local $L^{2}$ error has triangle markers. The dotted reference lines have slope 1,2 from top to bottom in the left graphic and 1,2,3 from top to bottom in the right graphic 

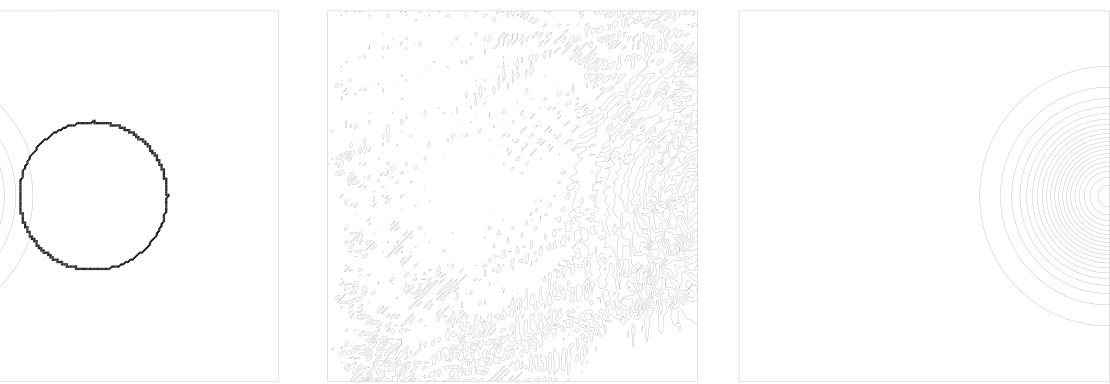

Fig. 5 From left to right: initial data on fine mesh, unstabilized solution, stabilized solution (nele $=80$, final time $t=1$ )

radius $r=0.2$ centered in the middle of the square and a Gaussian centered on the left boundary (see Fig. 5, left plot). The exact shapes are the same as those of the previous example. The solution is approximated over the time interval $(0,1]$ so that the cylinder leaves the domain at $t=0.7$ and at $t=1$ the Gaussian is centered on the right boundary. The time dependent inflow boundary condition $u=g$ on $\Gamma_{-}$is imposed weakly as described in (5.23) ( $g$ is chosen as the trace of the known exact solution). In Fig. 5, the final time approximation is reported in the middle plot without stabilization and the in right plot with stabilization. Observe that from $t=0.7$ the solution is smooth. Nevertheless the unstabilized Galerkin method fails to produce an accurate approximation of the smooth final time solution. Spurious oscillations from the discontinuity have spread over the whole computational domain and remain also when the rough part of the solution has left. The convergence of the $L^{2}$-error at final times for the stabilized and unstabilized approaches is shown in Fig. $6(h=1 /$ nele, nele $=40,80,160,320)$. We see that for the stabilized method both
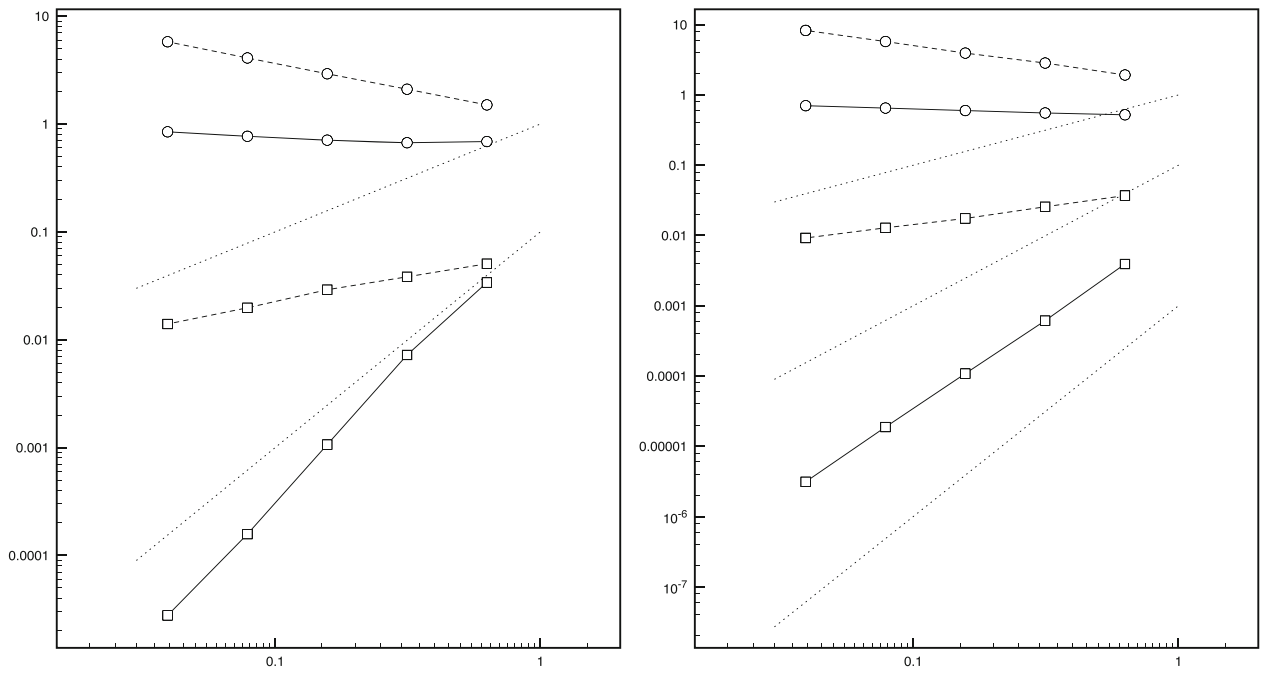

Fig. 6 Comparison of errors plotted against mesh size $h$ for stabilized (full line) and unstabilized (dashed line) methods with $P_{1}$ (left) and $P_{2}$ (right). Initial data from Fig. 5 (left plot). The space time error in material derivative has circle markers. The final time global $L^{2}$-error has square marker. The dotted reference lines have slope 1,2 from top to bottom in the left graphic and 1,2,3 from top to bottom in the right graphic 

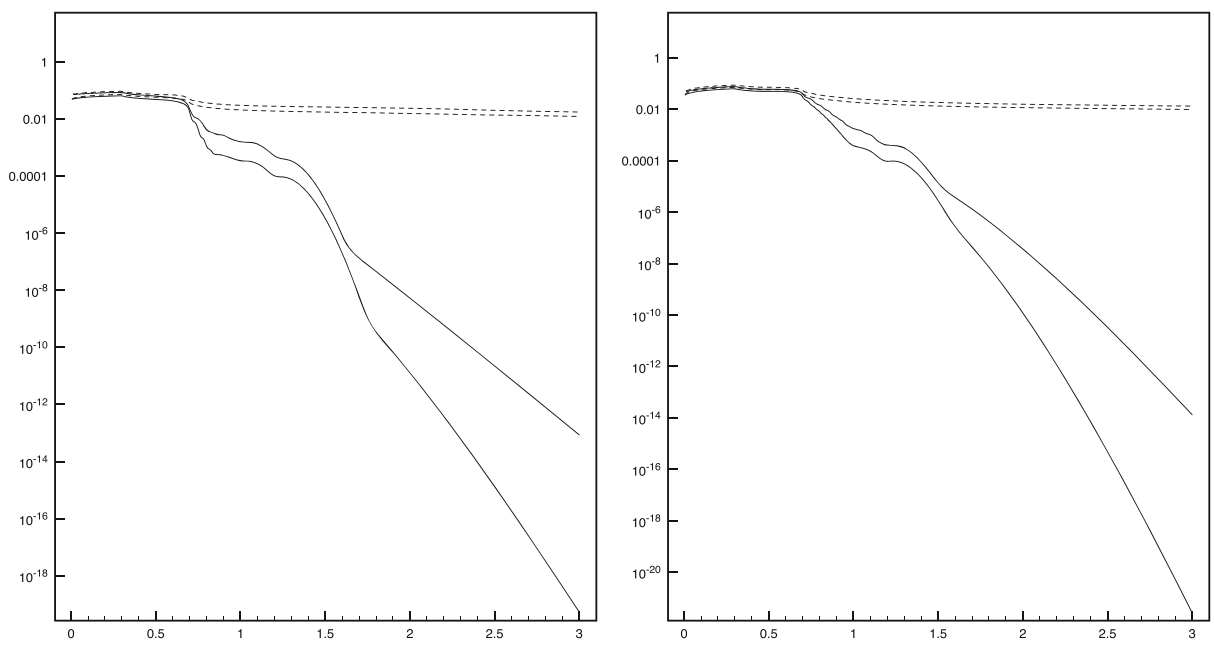

Fig. 7 Comparison of stabilized (full line) and unstabilized (dashed line) methods with $P_{1}$ (left) and $P_{2}$ (right) approximation. Evolution of the global $L^{2}$-error in time. Initial data from Fig. 5, left graphic. In each case the upper curve has nele $=40$ and the lower curve nele $=80$

the $P_{1}$ and $P_{2}$ approximations have optimal convergence to the smooth solution. The unstabilized method converges approximately as $O\left(h^{\frac{1}{2}}\right)$ in both cases and its material derivative diverges.

\subsection{Long Term Stability}

To see the effect of perturbations on the solution for long time we revisit the computational example of the previous section, but extend the time interval to $(0,3)$. The cylinder leaves the domain at $t=0.7$ and at the final time the solution is very small. One would then expect the error of the method to go to zero with machine precision, since the solution to approximate is very close to the trivial zero solution. In Fig. 7 the global $L^{2}$-norm is reported, for two consecutive meshes ( ele $=40$ and nele $=80$ ) and both the stabilized (full line) and the unstabilized (dashed line) methods. In the stabilized case the improvement of the approximation at $t=0.7$, when the cylinder leaves the domain, is clearly visible and the solution also improves as the Gaussian is evacuated. We see convergence to zero at machine precision of the error and also convergence under mesh refinement. In the unstabilized case the change at time $t=0.7$ is barely visible, the error decreases only very slowly in time and not noticeably under mesh refinement. Similarly as in the previous example, we conclude that the standard Galerkin method with weakly imposed boundary conditions in our simulations fails to evacuate the high frequency perturbations produced by the discontinuous initial data on the two meshes considered.

\section{Appendix}

Here we give the proofs of the approximation results for the $L^{2}$-projection, Lemmas 2 and 3 and finally the weighted discrete interpolation result (5.4). 
First we give a simple super approximation result for the Lagrange interpolant $i_{h}$ that will be useful for the proofs of inequalities (5.11) and (5.12). For a general discussion of discrete commutator properties we refer to [1].

Lemma 5 Let $\phi \in W^{k+1, \infty}(\Omega)$ satisfying (5.1) with $K>1$ and $h<1$. Then for $h^{\frac{1}{2}} / K$ sufficiently small, there holds for all $v_{h} \in V_{h}, S \in \mathcal{T}$,

$$
\left|\phi v_{h}-i_{h}\left(\phi v_{h}\right)\right|_{H^{s}(S)} \leq C h^{\frac{1}{2}-s} / K\left\|\phi v_{h}\right\|_{S}, \quad 0 \leq s \leq 2 .
$$

Proof By the approximation properties of $i_{h}$ there holds

$$
\left|\phi v_{h}-i_{h}\left(\phi v_{h}\right)\right|_{H^{s}(S)} \leq C h^{k+1-s}\left\|D^{k+1}\left(\phi v_{h}\right)\right\|_{S} .
$$

Using the product rule and the fact that $D^{k+1} v_{h}=0$ since $\left.v_{h}\right|_{S} \in \mathbb{P}_{k}(S)$, we see that

$$
\left\|D^{k+1}\left(\phi v_{h}\right)\right\|_{S} \leq C \sum_{l=1}^{k+1}|\phi|_{W^{l, \infty}(S)}\left|v_{h}\right|_{H^{k+1-l}(S)} .
$$

By applying the inverse inequality (2.6) repeatedly the derivatives on $v_{h}$ can be eliminated at the price of factors of the inverse of $h$,

$$
h^{k+1-s}\left\|D^{k+1}\left(\phi v_{h}\right)\right\|_{S} \leq C h^{1-s}\left\|v_{h}\right\|_{S} \sum_{l=1}^{k+1} h^{l-1}|\phi|_{W^{l, \infty}(S)} .
$$

Using the bound (5.1) it then follows that

$$
\sum_{l=1}^{k+1} h^{l-1}|\phi|_{W^{l, \infty}(S)} \leq C \sum_{l=1}^{k+1} h^{l-1}\left(K h^{\frac{1}{2}}\right)^{-l}\|\phi\|_{L^{\infty}(S)} \leq C\left(K h^{\frac{1}{2}}\right)^{-1}\|\phi\|_{L^{\infty}(S)} .
$$

Where we used the assumption that $h<1$ and $K>1$ in the last inequality. Combining the bounds (6.2), (6.3) and (6.4) it follows that

$$
\left\|\phi v_{h}-i_{h}\left(\phi v_{h}\right)\right\|_{H^{s}(S)} \leq C h^{1-s}\left(K h^{\frac{1}{2}}\right)^{-1}\|\phi\|_{L^{\infty}(S)}\left\|v_{h}\right\|_{S} .
$$

The claim now follows by applying (5.8).

Proof (Lemma 2) First note that by the construction of $\varpi$ there holds

$$
|\nabla \varpi| \leq C(\sqrt{h} K)^{-1} \varpi \leq(C \sqrt{h} / K) h^{-1} \varpi
$$

and we see that we may apply (5.5)-(5.7) with $\phi=\varpi$ for $(C \sqrt{h} / K)$ small enough.

Proof of (5.8). To prove (5.8), consider a triangle $S$, assume that the max value in $\max _{(x, t) \in S \times I_{\delta}} \varpi(x, t)$ is taken at $\left(x^{*}, t^{*}\right) \in S \times I_{\delta}$. Then

$$
\begin{aligned}
\max _{(x, t) \in S \times I_{\delta}} \varpi(x, t)\|v\|_{S}=\left\|\varpi\left(x^{*}, t^{*}\right) v\right\|_{S} & \leq\left\|\left(\varpi\left(x^{*}, t^{*}\right)-\varpi(\cdot, \tilde{t})\right) v\right\|_{S}+\|\varpi v\|_{S} \\
& \leq C h^{\frac{1}{2}} K^{-1} \varpi\left(x^{*}, t^{*}\right)\|v\|_{S}+\|\varpi(\cdot, \tilde{t}) v\|_{S},
\end{aligned}
$$

for any $\tilde{t} \in I_{\delta}$. Assuming that $C h^{\frac{1}{2}} K^{-1} \leq \frac{1}{2}$ we see that

$$
\max _{(x, t) \in S \times I_{\delta}} \varpi(x, t)\|v\|_{S} \leq 2\|\varpi(\cdot, \tilde{t}) v\|_{S}, \quad \forall \tilde{t} \in I_{\delta} .
$$


Proof of (5.9). For the proof of (5.9) first apply the stabilities (5.5)-(5.6). For the $L^{2}$-norm this yields

$$
\left\|\varpi\left(v-\pi_{h} v\right)\right\|_{\Omega} \leq\left\|\varpi\left(v-i_{h} v\right)\right\|_{\Omega}+\left\|\varpi \pi_{h}\left(i_{h} v-v\right)\right\|_{\Omega} \leq C\left\|\varpi\left(v-i_{h} v\right)\right\|_{\Omega} .
$$

Then apply interpolation locally and (5.8).

$$
\begin{aligned}
\left\|\varpi\left(v-i_{h} v\right)\right\|_{S} \leq \max _{x \in S} \varpi(x)\left\|v-i_{h} v\right\|_{S} & \leq C \max _{x \in S} \varpi(x) h^{k+1}\left\|D^{k+1} v\right\|_{S} \\
& \leq 2 C h^{k+1}\left\|\varpi D^{k+1} v\right\|_{S} .
\end{aligned}
$$

The claim follows by summing over $S \in \mathcal{T}$. The bound on the $H^{1}$-norm is identical.

Proof of (5.10). The stabilization operator is defined by the sum of the jumps of the gradient over the faces of the element. The first step is to split that jump using the triangle inequality over each face. Given a face $F=\partial S_{1} \cap \partial S_{2}$ for elements $S_{1}$ and $S_{2}$ this takes the form.

$$
\left\|\left[\nabla\left(v-\pi_{h} v\right)\right]\right\|_{F}^{2} \leq 2\left(\left\|\nabla\left(v-\pi_{h} v\right)\right\|_{\partial S_{1} \cap F}^{2}+\left\|\nabla\left(v-\pi_{h} v\right)\right\|_{\partial S_{2} \cap F}^{2}\right) .
$$

By breaking up the jumps on the contributions from respective element faces in this was we have

$$
s_{\varpi}\left(v-\pi_{h} v, v-\pi_{h} v\right) \leq C \sum_{S \in \mathcal{T}}\left(\max _{x \in S} \varpi(x)\right)^{2} h^{2} \beta_{\infty}\left\|\nabla\left(v-\pi_{h} v\right)\right\|_{\partial S}^{2} .
$$

Now apply the trace inequality (2.10) on each element to see that

$$
\left\|\nabla\left(v-\pi_{h} v\right)\right\|_{\partial S} \leq C\left(h^{\frac{1}{2}}\left|\nabla\left(v-\pi_{h} v\right)\right|_{H^{1}(S)}+h^{-\frac{1}{2}}\left\|\nabla\left(v-\pi_{h} v\right)\right\|_{S}\right) .
$$

For the first term in the right hand side add and subtract $i_{h} u$, split it using a triangle inequality and use an inverse inequality in one of the terms and interpolation in the other to see that

$$
\begin{aligned}
\left|\nabla\left(v-\pi_{h} v\right)\right|_{H^{1}(S)} & \leq C\left(\left|\nabla\left(v-i_{h} v\right)\right|_{H^{1}(S)}+\left|\nabla\left(i_{h} v-\pi_{h} v\right)\right|_{H^{1}(S)}\right) \\
& \leq C h^{k-1}\left\|D^{k+1} v\right\|_{S}+C h^{-1}\left\|\nabla\left(v-\pi_{h} v\right)\right\|_{S} .
\end{aligned}
$$

It follows using (5.8) that

$$
\sum_{S \in \mathcal{T}} \varpi(x)^{2} h^{2} \beta_{\infty}\left\|\nabla\left(v-\pi_{h} v\right)\right\|_{\partial S}^{2} \leq C \beta_{\infty} h^{2 k+1}\left\|D^{k+1} v\right\|_{\varpi}^{2}+C \beta_{\infty} h\left\|\nabla\left(v-\pi_{h} v\right)\right\|_{\varpi}^{2} .
$$

The claim now follows by applying (5.9) to the second term of the right hand side.

\section{Proof (Lemma 3)}

Proof of (5.11). To prove (5.11) recall that

$$
\left|\nabla \varpi^{-1}\right|=\left|\varpi^{-2} \nabla \varpi\right| \leq C(\sqrt{h} K)^{-1} \varpi^{-1}
$$

and we may apply (5.5) with $\phi=\varpi^{-1}$ to get

$$
\left\|\varpi^{-1}\left(\varpi^{2} v_{h}-\pi_{h}\left(\varpi^{2} v_{h}\right)\right)\right\|_{\Omega} \leq C\left\|\varpi^{-1}\left(\varpi^{2} v_{h}-i_{h}\left(\varpi^{2} v_{h}\right)\right)\right\|_{\Omega} .
$$

Consider one simplex $S$, take out the weight and then apply Lemma 5 followed by (5.8)

$$
\left\|\varpi^{-1}\left(\varpi^{2} v_{h}-i_{h}\left(\varpi^{2} v_{h}\right)\right)\right\|_{S} \leq\left(\max _{x \in S} \varpi^{-1}\right)\left\|\varpi^{2} v_{h}-i_{h}\left(\varpi^{2} v_{h}\right)\right\|_{S} \leq C h^{\frac{1}{2}} / K\left\|\varpi v_{h}\right\|_{S} .
$$


Finally take the square of both sides and sum over the simplices. The $H^{1}$-norm estimate follows using similar arguments.

Proof of (5.12). For the inequality (5.12) we consider one element of the sum and apply the trace inequality (2.10),

$$
\begin{aligned}
\left\|\varpi^{-1} \nabla\left(\varpi^{2} v_{h}-\pi_{h}\left(\varpi^{2} v_{h}\right)\right)\right\|_{\partial S} \leq & C\left(\max _{x \in S} \varpi^{-1} h^{\frac{1}{2}}\left|\nabla\left(\varpi^{2} v_{h}-\pi_{h}\left(\varpi^{2} v_{h}\right)\right)\right|_{H^{1}(S)}\right. \\
& \left.+\max _{x \in S} \varpi^{-1} h^{-\frac{1}{2}}\left\|\nabla\left(\varpi^{2} v_{h}-\pi_{h}\left(\varpi^{2} v_{h}\right)\right)\right\|_{S}\right) .
\end{aligned}
$$

In the first term, add and subtract $\nabla i_{h}\left(\varpi^{2} v_{h}\right)$ and use the triangle inequality followed by an inverse inequality to obtain

$$
\begin{aligned}
& \max _{x \in S} \varpi^{-1} h^{\frac{1}{2}}\left|\nabla\left(\varpi^{2} v_{h}-\pi_{h}\left(\varpi^{2} v_{h}\right)\right)\right|_{H^{1}(S)} \\
& \quad \leq C \max _{x \in S} \varpi^{-1} h^{\frac{1}{2}}\left(\left|\nabla\left(\varpi^{2} v_{h}-i_{h}\left(\varpi^{2} v_{h}\right)\right)\right|_{H^{1}(S)}+h^{-1}\left\|\nabla\left(i_{h} \varpi^{2} v_{h}-\pi_{h}\left(\varpi^{2} v_{h}\right)\right)\right\|_{S}\right) .
\end{aligned}
$$

For the first term in the right hand side we use Lemma 5, with $s=2$,

$$
\begin{aligned}
h^{\frac{1}{2}} \max _{x \in S} \varpi^{-1}\left|\nabla\left(\varpi^{2} v_{h}-i_{h}\left(\varpi^{2} v_{h}\right)\right)\right|_{H^{1}(S)} & \leq C \max _{x \in S} \varpi^{-1} K^{-1} h^{-1}\left\|\varpi^{2} v_{h}\right\|_{S} \\
& \leq C K^{-1} h^{-1}\left\|\varpi v_{h}\right\|_{S} .
\end{aligned}
$$

To bound the second term we use (5.8), sum over $S \in \mathcal{T}$ and use the stability of the $L^{2}$-projection (5.6) to get

$$
\sum_{S \in \mathcal{T}}\left(\max _{x \in S} \varpi^{-2}\right) h^{-1}\left\|\nabla\left(i_{h} \varpi^{2} v_{h}-\pi_{h}\left(\varpi^{2} v_{h}\right)\right)\right\|_{S}^{2} \leq C h^{-1}\left\|\varpi^{-1} \nabla\left(i_{h} \varpi^{2} v_{h}-\varpi^{2} v_{h}\right)\right\|_{\Omega}^{2} .
$$

We see that after summation over $S$ the second term in the right hand side of (6.5) also is on this form.

On every $S$ take out the factor $\max _{x \in S} \varpi^{-1}$ and apply Lemma 5 followed by (5.8) to arrive at

$$
h^{-\frac{1}{2}}\left\|\varpi^{-1} \nabla\left(i_{h} \varpi^{2} v_{h}-\varpi^{2} v_{h}\right)\right\|_{\Omega} \leq C K^{-1} h^{-1}\left\|\varpi v_{h}\right\|_{\Omega}
$$

which together with (6.6), summed over $S$, concludes the proof of (5.12).

Proof (Inequality (5.4)). For simplicity consider the form $\beta \cdot \nabla u_{h}=\partial_{x} u_{h}$. Using the product rule $\partial_{x}\left(\varpi^{2} v_{h}\right)=\left(\partial_{x} \varpi^{2}\right) v_{h}+\varpi^{2} \partial_{x} v_{h}$ and the triangle inequality it follows that

$$
\begin{aligned}
\left\|h^{\frac{1}{2}}\left(\partial_{x}\left(\varpi^{2} v_{h}\right)-\pi_{h}\left(\partial_{x}\left(\varpi^{2} v_{h}\right)\right)\right)\right\|_{\varpi^{-1}}^{2} \leq & 2 h\left\|\left(\partial_{x} \varpi^{2}\right) v_{h}-\pi_{h}\left(\partial_{x} \varpi^{2} v_{h}\right)\right\|_{\varpi^{-1}}^{2} \\
& +2 h\left\|\left(\varpi^{2} \partial_{x} v_{h}\right)-\pi_{h}\left(\varpi^{2} \partial_{x} v_{h}\right)\right\|_{\varpi^{-1}}^{2} .
\end{aligned}
$$

Noting that by the $L^{2}$-stability of $\pi_{h}$, the bound of $\varpi$, Lemma 5, (5.1) and (5.8)

$$
h\left\|\left(\partial_{x} \varpi^{2}\right) v_{h}-\pi_{h}\left(\partial_{x} \varpi^{2} v_{h}\right)\right\|_{\varpi^{-1}}^{2} \leq C h\left\|\left(\partial_{x} \varpi^{2}\right) v_{h}-i_{h}\left(\partial_{x} \varpi^{2} v_{h}\right)\right\|_{\varpi^{-1}}^{2} \leq C K^{-2}\left\|v_{h}\right\|_{\varpi}^{2} .
$$

It only remains to bound the second term of (6.7). We add and subtract $\pi_{0} \varpi^{2}$ defined by

$$
\left.\pi_{0} \varpi^{2}\right|_{S}=|S|^{-1} \int_{S} \varpi^{2}
$$


and use the triangle inequality to obtain

$$
\begin{aligned}
h\left\|\left(\varpi^{2} \partial_{x} v_{h}\right)-\pi_{h}\left(\varpi^{2} \partial_{x} v_{h}\right)\right\|_{\varpi^{-1}}^{2} \leq & C h\left\|\left(\varpi^{2} \partial_{x} v_{h}-\left(\pi_{0} \varpi^{2}\right) \partial_{x} v_{h}\right)\right\|_{\varpi^{-1}}^{2} \\
& +C h \|\left(\left(\pi_{0} \varpi^{2}\right) \partial_{x} v_{h}-\pi_{h}\left(\left(\pi_{0} \varpi^{2}\right) \partial_{x} v_{h}\right) \|_{\varpi^{-1}}^{2}\right. \\
& +C h\left\|\left(\pi_{h}\left(\left(\pi_{0} \varpi^{2}\right) \partial_{x} v_{h}\right)-\pi_{h}\left(\varpi^{2} \partial_{x} v_{h}\right)\right)\right\|_{\varpi^{-1}}^{2} \\
= & T_{1}+T_{2}+T_{3} .
\end{aligned}
$$

First, for $T_{3}$, observe that by the stability of the $L^{2}$-projection (5.5) we have

$$
h\left\|\left(\pi_{h}\left(\left(\pi_{0} \varpi^{2}\right) \partial_{x} v_{h}\right)-\pi_{h}\left(\varpi^{2} \partial_{x} v_{h}\right)\right)\right\|_{\varpi^{-1}}^{2} \leq C h\left\|\left(\varpi^{2} \partial_{x} v_{h}-\left(\pi_{0} \varpi^{2}\right) \partial_{x} v_{h}\right)\right\|_{\varpi^{-1}}^{2} \leq C T_{1},
$$

so only $T_{1}$ and $T_{2}$ need to be bounded. For $T_{1}$, by the approximation $\left\|\varpi^{2}-\pi_{0} \varpi^{2}\right\|_{L^{\infty}(S)} \leq$ $C h^{\frac{1}{2}} / K\|\varpi\|_{L^{\infty}(S)}^{2}$ and applying (5.8) repeatedly, we have for one simplex $S$,

$$
\begin{aligned}
\left\|\varpi^{-1}\left(\varpi^{2} \partial_{x} v_{h}-\left(\pi_{0} \varpi^{2}\right) \partial_{x} v_{h}\right)\right\|_{S} & \leq h^{\frac{1}{2}} / K \max _{x \in S} \varpi^{2} \max _{x \in S} \varpi^{-1}\left\|\partial_{x} v_{h}\right\|_{S} \\
& \leq C h^{-\frac{1}{2}} K^{-1}\left\|\varpi v_{h}\right\|_{S} .
\end{aligned}
$$

Taking the square of both sides and summing over all simplices yields the bound for $T_{1}$,

$$
h\left\|\left(\varpi^{2} \partial_{x} v_{h}-\left(\pi_{0} \varpi^{2}\right) \partial_{x} v_{h}\right)\right\|_{\varpi^{-1}}^{2} \leq C K^{-2}\left\|v_{h}\right\|_{\varpi}^{2} .
$$

Finally for the term $T_{2}$ we use (5.3) with $\boldsymbol{\beta}_{0}=\left(\pi_{0} \varpi^{2}\right) e_{x}$. This leads to

$$
h \|\left(\left(\pi_{0} \varpi^{2}\right) \partial_{x} v_{h}-\pi_{h}\left(\left(\pi_{0} \varpi^{2}\right) \partial_{x} v_{h}\right) \|_{\varpi^{-1}}^{2} \leq C_{w s} s_{\varpi^{-1}}\left(\left(\pi_{0} \varpi^{2}\right) v_{h},\left(\pi_{0} \varpi^{2}\right) v_{h}\right) .\right.
$$

Adding and subtracting $\varpi^{2}$ and using the triangle inequality and the fact that $\varpi^{2}$ is smooth leads to

$s_{\varpi^{-1}}\left(\left(\pi_{0} \varpi^{2}\right) v_{h},\left(\pi_{0} \varpi^{2}\right) v_{h}\right) \leq 2 s_{\varpi}\left(v_{h}, v_{h}\right)+2 s_{\varpi^{-1}}\left(\left(\varpi^{2}-\pi_{0} \varpi^{2}\right) v_{h},\left(\varpi^{2}-\pi_{0} \varpi^{2}\right) v_{h}\right)$.

For the second term of the right hand side consider the boundary of one triangle and apply the trace inequality (2.10), followed by the approximation of $\pi_{0}$ to get

$$
\begin{aligned}
\left\|h\left(\varpi^{2}-\pi_{0} \varpi^{2}\right) \nabla v_{h}\right\|_{\partial S} & \leq C \max _{x \in S} \varpi^{2} K^{-1} h^{\frac{3}{2}}\left(h^{\frac{1}{2}}\left|\nabla v_{h}\right|_{H^{1}(S)}+h^{-\frac{1}{2}}\left\|\nabla v_{h}\right\|_{S}\right) \\
& \leq C K^{-1}\left\|\varpi^{2} v_{h}\right\|_{S} .
\end{aligned}
$$

The last step followed using the inverse inequality (2.6) and (5.8). Proceeding by applying the previous bound to all triangle faces, it follows that

$$
\begin{aligned}
& s_{\varpi^{-1}}\left(\left(\varpi^{2}-\pi_{0} \varpi^{2}\right) v_{h},\left(\varpi^{2}-\pi_{0} \varpi^{2}\right) v_{h}\right) \leq C \sum_{S \in \mathcal{T}} \max _{x \in S} \varpi^{-2}\left\|h\left(\varpi^{2}-\pi_{0} \varpi^{2}\right) \nabla v_{h}\right\|_{\partial S}^{2} \\
& \leq C \sum_{S \in \mathcal{T}} \max _{x \in S} \varpi^{-2} K^{-2}\left\|\varpi^{2} v_{h}\right\|_{S}^{2} \leq C K^{-2}\left\|v_{h}\right\|_{\varpi}^{2},
\end{aligned}
$$

where the last step follows using (5.8). The proof is now finished by collecting the bounds (6.8)-(6.9). 
Proof (Lemma 4). Using $\delta t \leq C h$ and (5.1) there holds

$$
\begin{aligned}
\left\|v_{h} \int_{t_{n-1}}^{t_{n}} \partial_{t} \varpi \mathrm{d} t\right\|_{\Omega} & \leq C \delta t /\left(K h^{\frac{1}{2}}\right)\left(\sum_{S \in \mathcal{T}} \max _{(x, t) \in S \times\left[t_{n-1}, t_{n}\right]} \varpi(x, t)^{2}\left\|v_{h}\right\|_{S}^{2}\right)^{\frac{1}{2}} \\
& \leq \delta t^{\frac{1}{2}} C / K\left\|v_{h}\right\|_{\varpi_{n}} .
\end{aligned}
$$

For the second inequality we applied (5.8) elementwise and then upper bounded $\min _{t \in\left[t_{n-1}, t_{n}\right]}\left\|v_{h} \varpi(\cdot, t)\right\|_{S}$ by $\left\|v_{h}\right\|_{\varpi_{n}}$. For the bound of the second term observe that, estimating

$$
\left|\int_{t_{n-1}}^{t_{n}} \int_{t}^{t_{n}} \partial_{t t} \varpi^{2} \mathrm{~d} s \mathrm{~d} t\right| \leq \delta t^{2} \max _{t \in\left[t_{n-1}, t_{n}\right]}\left|\partial_{t t} \varpi^{2}\right|
$$

and then applying (5.1) repeatedly with $l=1$ and 2 , to show

$$
\max _{t \in\left[t_{n-1}, t_{n}\right]}\left|\partial_{t t} \varpi^{2}\right| \leq C^{2} h^{-1} K^{-2} \max _{t \in\left[t_{n-1}, t_{n}\right]} \varpi^{2} .
$$

It follows that for all $S \in \mathcal{T}$,

$$
\left\|v_{h}\left|\int_{t_{n-1}}^{t_{n}} \int_{t}^{t_{n}} \partial_{t t} \varpi^{2} \mathrm{~d} s \mathrm{~d} t\right|^{\frac{1}{2}}\right\|_{S} \leq \delta t^{\frac{1}{2}} C K^{-1} \max _{(x, t) \in S \times\left[t_{n-1}, t_{n}\right]} \varpi\left\|v_{h}\right\|_{S} .
$$

Applying (5.8) we conclude that

$$
\begin{aligned}
\sum_{S \in \mathcal{T}}\left\|v_{h}\left|\int_{t_{n-1}}^{t_{n}} \int_{t}^{t_{n}} \partial_{t t} \varpi^{2} \mathrm{~d} s \mathrm{~d} t\right|^{\frac{1}{2}}\right\|_{S}^{2} & \leq \delta t C^{2} K^{-2} \sum_{S \in \mathcal{T}} \min _{t \in\left[t_{n-1}, t_{n}\right]}\left\|v_{h} \varpi(\cdot, t)\right\|_{S}^{2} \\
& \leq \delta t C^{2} K^{-2}\left\|v_{h}\right\|_{\varpi_{n}}^{2} .
\end{aligned}
$$

Funding The author acknowledges funding from EPSRC grants EP/P01576X/1 and EP/T033126/1.

Availability of data and material The data used to produce figures can be made available upon reasonable request.

Code Availability Codes used to produce approximate solutions can be made available upon reasonable request.

Open Access This article is licensed under a Creative Commons Attribution 4.0 International License, which permits use, sharing, adaptation, distribution and reproduction in any medium or format, as long as you give appropriate credit to the original author(s) and the source, provide a link to the Creative Commons licence, and indicate if changes were made. The images or other third party material in this article are included in the article's Creative Commons licence, unless indicated otherwise in a credit line to the material. If material is not included in the article's Creative Commons licence and your intended use is not permitted by statutory regulation or exceeds the permitted use, you will need to obtain permission directly from the copyright holder. To view a copy of this licence, visit http://creativecommons.org/licenses/by/4.0/.

\section{References}

1. Bertoluzza, S.: The discrete commutator property of approximation spaces. C. R. Acad. Sci. Paris Sr. I Math. 329, 1097-1102 (1999)

2. Boman, M.: Estimates for the $l_{2}$-projection onto continuous finite element spaces in a weighted $l_{p}$-norm. BIT Number. Math. 46, 249-260 (2006) 
3. Brenner, S.C., Scott, L.R.: The Mathematical Theory of Finite Element Methods, 3rd edn. Texts in Applied Mathematics, vol. 15. Springer, New York (2008)

4. Brezzi, F., Marini, L.D., Süli, E.: Discontinuous Galerkin methods for first-order hyperbolic problems. Math. Models Methods Appl. Sci. 14, 1893-1903 (2004)

5. Burman, E., Gillissen, J., Oksanen, L.: Stability estimate for scalar image velocimetry. arXiv:2008.09451 (2020)

6. Burman, E., Stamm, B.: Minimal stabilization for discontinuous Galerkin finite element methods for hyperbolic problems. J. Sci. Comput. 33, 183-208 (2007)

7. Burman, E.: A unified analysis for conforming and nonconforming stabilized finite element methods using interior penalty. SIAM J. Numer. Anal. 43, 2012-2033 (2005)

8. Burman, E.: A posteriori error estimation for interior penalty finite element approximations of the advection-reaction equation. SIAM J. Numer. Anal. 47, 3584-3607 (2009)

9. Burman, E.: Robust error estimates in weak norms for advection dominated transport problems with rough data. Math. Models Methods Appl. Sci. 24, 2663-2684 (2014)

10. Burman, E., Ern, A.: Continuous interior penalty $h p$-finite element methods for advection and advectiondiffusion equations. Math. Comput. 76, 1119-1140 (2007)

11. Burman, E., Ern, A.: Implicit-explicit Runge-Kutta schemes and finite elements with symmetric stabilization for advection-diffusion equations. ESAIM Math. Model. Numer. Anal. 46, 681-707 (2012)

12. Burman, E., Ern, A., Fernández, M.A.: Explicit Runge-Kutta schemes and finite elements with symmetric stabilization for first-order linear PDE systems. SIAM J. Numer. Anal. 48, 2019-2042 (2010)

13. Burman, E., Guzmán, J.: Implicit-explicit multistep formulations for finite element discretisations using continuous interior penalty. arXiv:2012.05727. ESAIM Math. Model. Numer. Anal. (to appear) (2020)

14. Burman, E., Guzmán, J., Leykekhman, D.: Weighted error estimates of the continuous interior penalty method for singularly perturbed problems. IMA J. Numer. Anal. 29, 284-314 (2009)

15. Burman, E., Hansbo, P.: Edge stabilization for Galerkin approximations of convection-diffusionreaction problems. Comput. Methods Appl. Mech. Eng. 193, 1437-1453 (2004)

16. Burman, E., Nechita, M., Oksanen, L.: A stabilized finite element method for inverse problems subject to the convection-diffusion equation, II: convection-dominated regime. arXiv:2006.13201 (2020)

17. Burman, E., Quarteroni, A., Stamm, B.: Stabilization strategies for high order methods for transport dominated problems. Boll. Unione Mat. Ital. Ser. (9) 1, 57-77 (2008)

18. Burman, E., Quarteroni, A., Stamm, B.: Interior penalty continuous and discontinuous finite element approximations of hyperbolic equations. J. Sci. Comput. 43, 293-312 (2010)

19. Moura, R.C., da Silva, A.F.C., Burman, E., Sherwin, S.J.: Eigenanalysis of gradient-jump penalty (GJP) stabilisation for CG. Technical report. https://doi.org/10.13140/RG.2.2.32887.85924 (2020)

20. de Frutos, J., García-Archilla, B., Novo, J.: Local error estimates for the SUPG method applied to evolutionary convection-reaction-diffusion equations. J. Sci. Comput. 66, 528-554 (2016)

21. Douglas, J., Dupont, T.: Interior penalty procedures for elliptic and parabolic Galerkin methods. In: Glowinski, R., Lions, J.L. (eds.) Computing Methods in Applied Sciences (Second International Symposium, Versailles, 1975). Lecture Notes in Physics, vol. 58, pp. 207-216. Springer, Berlin (1976)

22. Eriksson, K., Johnson, C.: Adaptive finite element methods for parabolic problems. II. Optimal error estimates in $l_{\infty} l_{2}$ and $l_{\infty} l_{\infty}$. SIAM J. Numer. Anal. 32, 706-740 (1995)

23. Ern, A., Guermond, J.-L.: Theory and Practice of Finite Elements. Applied Mathematical Sciences, vol. 159. Springer, New York (2004)

24. Ern, A., Guermond, J.-L.: Finite Elements, vol. III. Springer, Cham (2021)

25. Ghrist, M.L., Fornberg, B., Reeger, J.A.: Stability ordinates of Adams predictor-corrector methods. BIT Numer. Math. 55, 733-750 (2015)

26. Girault, V., Scott, L.R.: On a time-dependent transport equation in a Lipschitz domain. SIAM J. Math. Anal. 42, 1721-1731 (2010)

27. Guermond, J.-L.: Subgrid stabilization of Galerkin approximations of linear contraction semi-groups of class $c^{0}$ in Hilbert spaces. Numer. Methods Partial Differ. Equ. 17, 1-25 (2001)

28. Guzmán, J.: Local analysis of discontinuous Galerkin methods applied to singularly perturbed problems. J. Numer. Math. 14, 41-56 (2006)

29. Hecht, F.: New development in FreeFem++. J. Numer. Math. 20, 251-265 (2013)

30. Houston, P., Mackenzie, J.A., Süli, E., Warnecke, G.: A posteriori error analysis for numerical approximations of Friedrichs systems. Numer. Math. 82, 433-470 (1999)

31. Hundsdorfer, W., Verwer, J.: Numerical Solution of Time-Dependent Advection-Diffusion-Reaction Equations. Springer Series in Computational Mathematics, vol. 33. Springer, Berlin (2003)

32. Johnson, C., Schatz, A.H., Wahlbin, L.B.: Crosswind smear and pointwise errors in streamline diffusion finite element methods. Math. Comput. 49, 25-38 (1987) 
33. Johnson, C., Nävert, U., Pitkäranta, J.: Finite element methods for linear hyperbolic problems. Comput. Methods Appl. Mech. Eng. 45, 285-312 (1984)

34. Karamanos, G.-S., Karniadakis, G.E.: A spectral vanishing viscosity method for large-eddy simulations. J. Comput. Phys. 163, 22-50 (2000)

35. Maday, Y., Tadmor, E.: Analysis of the spectral vanishing viscosity method for periodic conservation laws. SIAM J. Numer. Anal. 26, 854-870 (1989)

36. Moura, R.C., Aman, M., Peiró, J., Sherwin, S.J.: Spatial eigenanalysis of spectral/hp continuous Galerkin schemes and their stabilisation via DG-mimicking spectral vanishing viscosity for high Reynolds number flows. J. Comput. Phys. 406, 109112 (2020)

37. Moura, R.C., Cassinelli, A., da Silva, A.F.C., Burman, E., Sherwin, S.J.: Gradient jump penalty stabilisation of spectral/hp element discretisation for under-resolved turbulence simulations. Comput. Methods Appl. Mech. Eng. 388, 114200 (2022)

38. Peterson, T.E., Shuster, D.B.: Non-optimal behaviour of finite element methods for first order hyperbolic problems. Appl. Math. Comput. Sci. 5, 579-596 (1995)

39. Wang, H., Liu, Y., Zhang, Q., Shu, C.-W.: Local discontinuous Galerkin methods with implicit-explicit time-marching for time-dependent incompressible fluid flow. Math. Comput. 88, 91-121 (2019)

40. Xu, Y., Shu, C.-W., Zhang, Q.: Error estimate of the fourth-order Runge-Kutta discontinuous Galerkin methods for linear hyperbolic equations. SIAM J. Numer. Anal. 58, 2885-2914 (2020)

41. Xu, Y., Zhang, Q., Shu, C.-W., Wang, H.: The $l^{2}$-norm stability analysis of Runge-Kutta discontinuous Galerkin methods for linear hyperbolic equations. SIAM J. Numer. Anal. 57, 1574-1601 (2019)

42. Zhang, Q., Shu, C.-W.: Error estimates to smooth solutions of Runge-Kutta discontinuous Galerkin methods for scalar conservation laws. SIAM J. Numer. Anal. 42, 641-666 (2004)

43. Zhang, Q., Shu, C.-W.: Stability analysis and a priori error estimates of the third order explicit RungeKutta discontinuous Galerkin method for scalar conservation laws. SIAM J. Numer. Anal. 48, 1038-1063 (2010)

44. Zhou, G.H.: A local $l^{2}$-error analysis of the streamline diffusion method for nonstationary convectiondiffusion systems. RAIRO Model. Math. Anal. Numér. 29, 577-603 (1995)

Publisher's Note Springer Nature remains neutral with regard to jurisdictional claims in published maps and institutional affiliations. 